\title{
Connecting Antarctic Cross-Slope Exchange with Southern Ocean Overturning
}

\author{
ANDREW L. STEWART AND ANDREW F. THOMPSON \\ Environmental Sciences and Engineering, California Institute of Technology, Pasadena, California
}

(Manuscript received 14 October 2012, in final form 11 March 2013)

\begin{abstract}
Previous idealized investigations of Southern Ocean overturning have omitted its connection with the Antarctic continental shelves, leaving the influence of shelf processes on Antarctic Bottom Water (AABW) export unconsidered. In particular, the contribution of mesoscale eddies to setting the stratification and overturning circulation in the Antarctic Circumpolar Current (ACC) is well established, yet their role in cross-shelf exchange of water masses remains unclear. This study proposes a residual-mean theory that elucidates the connection between Antarctic cross-shelf exchange and overturning in the ACC, and the contribution of mesoscale eddies to the export of AABW. The authors motivate and verify this theory using an eddy-resolving process model of a sector of the Southern Ocean. The strength and pattern of the simulated overturning circulation strongly resemble those of the real ocean and are closely captured by the residualmean theory. Over the continental slope baroclinic instability is suppressed, and so transport by mesoscale eddies is reduced. This suppression of the eddy fluxes also gives rise to the steep "V"-shaped isopycnals that characterize the Antarctic Slope Front in AABW-forming regions of the continental shelf. Furthermore, to produce water on the continental shelf that is dense enough to sink to the deep ocean, the deep overturning cell must be at least comparable in strength to wind-driven mean overturning on the continental slope. This results in a strong sensitivity of the deep overturning strength to changes in the polar easterly winds.
\end{abstract}

\section{Introduction}

The meridional overturning circulation (MOC) describes the global-scale meridional transport of ocean water masses (Talley et al. 2003). It exerts a substantial influence over climate via interhemispheric transport of heat and salt, in addition to biogeochemical tracers such as oxygen and carbon (Kuhlbrodt et al. 2007; LynchStieglitz et al. 2007). This article focuses on the Southern Ocean, which has been identified as a region of particular dynamical and climatic importance for the MOC (Marshall and Speer 2012). Here strong, midlatitude westerly winds produce an isopycnal tilt that allows deep waters to upwell to the surface. These waters are then transported north by surface currents and subducted as Antarctic Intermediate Water (AAIW) or transported south toward the Antarctic continental shelf (Speer et al. 2000). In certain regions, largely confined to the Weddell and Ross Seas, the upwelled water mixes with fresh

Corresponding author address: Andrew L. Stewart, Environmental Science and Engineering, MC 131-24, California Institute of Technology, Pasadena, CA 91125.

E-mail: stewart@gps.caltech.edu glacial meltwater, and high-salinity shelf water produced by rejection of brine during sea ice formation (Gill 1973; Orsi and Wiederwohl 2009). The resulting mixture sinks down from the continental shelf and spreads throughout the global ocean as Antarctic Bottom Water (AABW) (Gordon 2009).

The export of AABW constitutes the lowest branch of the global MOC and is arguably the most climatically important over millennial time scales (Marshall and Speer 2012). AABW consists largely of waters that have recently been in contact with the atmosphere and so carries oxygen to the deep ocean (Orsi et al. 2001). The deep branch of the MOC also stores dissolved $\mathrm{CO}_{2}$ and may thereby drive millennial-scale climate change via exchanges with the atmospheric $\mathrm{CO}_{2}$ reservoir (Russell et al. 2006; Toggweiler et al. 2006; Toggweiler 2009; Skinner et al. 2010).

The climatic influence of the Southern Ocean MOC has motivated a series of studies to elucidate the relationship between surface forcing of the Antarctic Circumpolar Current (ACC) and the resultant stratification and overturning (e.g., Hallberg and Gnanadesikan 2006; Hogg et al. 2008). Anthropogenic $\mathrm{CO}_{2}$ emissions have led to an intensification of the midlatitude westerlies in 
the past few decades (Shindell and Schmidt 2004; Polvani et al. 2011), characterized by a strengthening of the southern annular mode (Thompson and Wallace 2000; Thompson et al. 2000). Meanwhile, millennial-scale $\mathrm{CO}_{2}$ exchanges between the deep ocean and the atmosphere have also been attributed to latitudinal shifts of the westerlies (Russell et al. 2006; Toggweiler et al. 2006; Toggweiler 2009). This has motivated a particular focus on the response of the MOC to changes in the midlatitude westerly wind strength (e.g., Hofmann and Maqueda 2011; Meredith et al. 2011; Abernathey et al. 2011).

The upper branch of the Southern Ocean MOC may be explained dynamically using residual-mean theory (RMT) (Andrews et al. 1987; Plumb and Ferrari 2005; Kuo et al. 2005). The observed overturning is described as a relatively small residual, resulting from an imbalance between a wind-driven mean overturning and a counteracting "eddy" overturning (Marshall and Radko 2003; Olbers and Visbeck 2005; Marshall and Radko 2006; Radko and Marshall 2006). RMT qualitatively explains the observed tracer distributions in the Southern Ocean (Karsten and Marshall 2002) and compares favorably with idealized eddy-resolving simulations (Abernathey et al. 2011). Wolfe and Cessi (2011) extended RMT to model the pole-to-pole MOC, and found good agreement with eddy-resolving simulations of an entire ocean basin. Ito and Marshall (2008) applied RMT to the deep MOC, and derived a scaling for the diapycnal mixing rate needed to allow transport of AABW across isopycnals outcropping from the ocean bed. Saenko et al. (2011) combined an extension of this scaling with eddypermitting simulations of the Southern Ocean to predict an increase in AABW export in response to strengthening of the midlatitude westerlies. Nikurashin and Vallis (2011) developed a residual-mean model of a diffusively driven deep overturning cell, and Nikurashin and Vallis (2012) extended this model to include a quasi-adiabatic upper cell originating from a northern high-latitude convective region. Their model agreed qualitatively with coarsely resolved simulations using parameterized eddy transports.

The present work employs RMT as a model of both the upper and lower overturning cells, including an idealized Antarctic continental shelf, in concert with idealized eddy-resolving simulations of a sector of the Southern Ocean. This connects our theoretical understanding of the MOC in the ACC with that of the Antarctic Slope Front (ASF), a weaker westward current that flows along the continental slope around most of Antarctica (Jacobs 1991; Muench and Gordon 1995; Chavanne et al. 2010). The ASF is driven by circumpolar easterly winds around the Antarctic margins (Large and
Yeager 2009), and is characterized by steep "V"'-shaped isopycnals in AABW-forming regions of the continental shelf (Gill 1973; Thompson and Heywood 2008). Our understanding of the dynamical processes that set the structure of the ASF is limited to quite idealized models. For example, Ou (2007) proposed a box-type model of the ASF that presupposed the "V"-shaped isopycnal structure and then balanced heat and salt fluxes across the continental slope. More recently Baines (2009) constructed more sophisticated analytical solutions for a two-dimensional flow that is entrained into a turbulent down-slope bottom current. In this article, the structure of the ASF is explained in section 5 as a consequence of the eddy-mean overturning balance of RMT, once the tendency of the continental slope to suppress baroclinic instability is accounted for.

Connecting the overturning in the ACC with that over the Antarctic continental shelf allows us to analyze the influence of shelf processes on the global MOC. In particular, the contribution of mesoscale eddies to the cross-shelf transport remains unclear, though recent high-resolution modeling studies suggest that eddies may dominate the shoreward transport of Circumpolar Deep Water (CDW) beneath Antarctic ice shelves (Thoma et al. 2008; Dinniman et al. 2011; Nøst et al. 2011). We will focus on the sensitivity of the MOC to changes in the easterlies that drive the ASF. While the midlatitude westerlies have intensified over the past few decades (Shindell and Schmidt 2004; Polvani et al. 2011), the trend in the polar easterlies is ambiguous (Son et al. 2010; Polvani et al. 2011), as are model predictions for their strength at the last glacial maximum (Krinner and Genthon 1998; Kitoh et al. 2001). Stewart and Thompson (2012) have recently shown that the export of $\mathrm{AABW}$ is much more sensitive to changes in the polar easterlies than the midlatitude westerlies. This sensitivity is explained succinctly by our RMT approach and is explored further in section 6.

The structure of this paper is as follows. In section 2 we describe our idealized eddy-resolving numerical simulations of the ACC and ASF and diagnose the MOC and cross-shelf heat transport. In section 3 we derive residual-mean equations and boundary equations suitable for our domain. In section 4 we discuss the parameterizations of the isopycnal and diapycnal diffusivities required to represent the heat transport over the Antarctic continental slope and close to the ocean bed. In section 5 we obtain approximate and numerical solutions to the residual-mean equations. Finally, in section 6 we examine the sensitivity of the stratification and overturning circulation to variations in the imposed wind stress, and compare the results of our theory with those of our eddy-resolving simulations. 
TABLE 1. List of parameters used in our reference simulation.

\begin{tabular}{|c|c|c|}
\hline Parameter & Value & Description \\
\hline$L_{x}$ & $1000 \mathrm{~km}$ & Zonal domain size \\
\hline$L_{y}$ & $2000 \mathrm{~km}$ & Meridional domain size \\
\hline$H$ & $3000 \mathrm{~km}$ & Maximum ocean depth \\
\hline$H_{s}$ & $500 \mathrm{~m}$ & $\begin{array}{l}\text { Ocean depth on the continental } \\
\text { shelf }\end{array}$ \\
\hline$Y_{s}$ & $500 \mathrm{~km}$ & Slope center position \\
\hline$W_{s}$ & $100 \mathrm{~km}$ & Slope half-width \\
\hline$L_{c}$ & $200 \mathrm{~km}$ & Radius of shelf cooling \\
\hline$T_{c}$ & 7 days & Shelf cooling time scale \\
\hline$L_{r}$ & $100 \mathrm{~km}$ & $\begin{array}{l}\text { Width of northern relaxation } \\
\text { region }\end{array}$ \\
\hline$T_{r}$ & 7 days & Northern relaxation time scale \\
\hline$L_{Q}$ & $1700 \mathrm{~km}$ & Width of thermal surface forcing \\
\hline$\rho_{0}$ & $999.8 \mathrm{~kg} \mathrm{~m}^{-3}$ & Reference density \\
\hline$\alpha$ & $2 \times 10^{-4} K^{-1}$ & Thermal expansion coefficient \\
\hline$g$ & $9.81 \mathrm{~m}^{2} \mathrm{~s}^{-1}$ & Gravitational constant \\
\hline$f_{0}$ & $-1 \times 10^{-4} \mathrm{~s}^{-1}$ & Reference Coriolis parameter $f$ \\
\hline$\beta$ & $10^{-11} \mathrm{~m}^{-1} \mathrm{~s}^{-1}$ & Meridional gradient of $f$ \\
\hline$\tau_{\mathrm{ACC}}, \tau_{\mathrm{ASF}}$ & $0.2,-0.05 \mathrm{~N} \mathrm{~m}^{-2}$ & Wind stress scales \\
\hline$Q_{0}$ & $10 \mathrm{~W} \mathrm{~m}^{-2}$ & Heat flux scale \\
\hline$\widetilde{A}_{h}$ & $12 \mathrm{~m}^{2} \mathrm{~s}^{-1}$ & Horizontal viscosity \\
\hline$A_{v}$ & $3 \times 10^{-4} \mathrm{~m}^{2} \mathrm{~s}^{-1}$ & Vertical viscosity \\
\hline$A_{4 \text { grid }}$ & 0.1 & $\begin{array}{l}\text { Grid-dependent biharmonic } \\
\text { viscosity }\end{array}$ \\
\hline$C_{4}$ leith & 1.0 & Leith vortical viscosity \\
\hline$C_{4}$ leithD & 1.0 & Leith solenoidal viscosity \\
\hline$\kappa_{v}$ & $5 \times 10^{-6} \mathrm{~m}^{2} \mathrm{~s}^{-1}$ & Vertical diffusivity \\
\hline$r_{b}$ & $1.1 \times 10^{-3} \mathrm{~m} \mathrm{~s}^{-1}$ & Bottom friction \\
\hline$\Delta_{x}, \Delta_{y}$ & $4.9,5.0 \mathrm{~km}$ & Horizontal grid spacing \\
\hline$\Delta_{z}$ & $12.5 \mathrm{~m}-187.5 \mathrm{~m}$ & Vertical grid spacing \\
\hline$\Delta_{t}$ & $566 \mathrm{~s}$ & Time step size \\
\hline
\end{tabular}

\section{Eddy-resolving simulations of the deep overturning circulation}

In this section we discuss the overturning circulation and export of dense water from the continental shelf in our reference simulation, whose imposed forcing most strongly resembles conditions in the contemporary Southern Ocean. This simulation motivates some aspects of the residual-mean theory described in sections $3-5$.

\section{a. Modeling preliminaries}

We used the Massachusetts Institute of Technology (MIT) general circulation model (MITgcm) to conduct all of the simulations described herein (Marshall et al. 1997a,b). The model parameters are summarized in Table 1. We model a sector of the Southern Ocean and the Antarctic continental shelf as a zonally reentrant channel in Cartesian geometry. The channel is zonally symmetric, and its depth is described by

$$
h(y)=H_{s}+\frac{1}{2}\left(H-H_{s}\right)\left[1+\tanh \left(\frac{y-Y_{s}}{W_{s}}\right)\right] .
$$

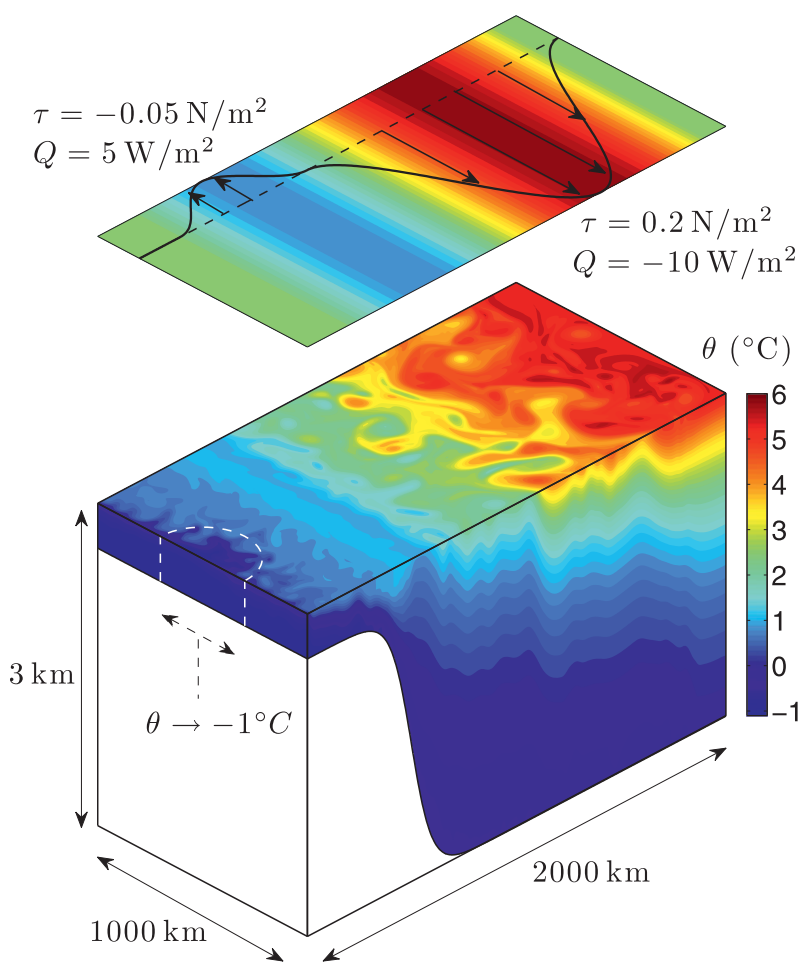

FIG. 1. Three-dimensional temperature snapshot from our reference simulation in statistically steady state. (top) The profiles of surface wind stress and heating/cooling are shown (note that $Q>0$ corresponds to heat loss to the atmosphere).

This geometry is illustrated in Fig. 1. For simplicity and computational efficiency we have used a somewhat shallower channel than the $\sim 4000$-m depth of the real Southern Ocean.

Flow in the channel is governed by the threedimensional, hydrostatic Boussinesq equations (e.g., Vallis 2006). We neglect the dynamical influence of salinity, which is fixed at 35 psu everywhere, and prescribe a linear dependence of density on temperature. Temperature is advected using a third-order direct spacetime flux-limiting scheme. We parameterize dense water formation by directly relaxing the temperature toward $\theta_{c}=-1^{\circ} \mathrm{C}$ over a localized area of the shelf. We choose a relaxation time scale profile that increases exponentially with horizontal distance from $x=y=0$,

$$
T(x, y, z)=T_{c} \frac{H_{s}}{|z|} \exp \left[\left(\frac{x}{1 / 2 L_{c}}\right)^{2}+\left(\frac{y}{1 / 2 L_{c}}\right)^{2}\right], \quad y \leq L_{c}
$$

where $T_{c}=7$ days and $L_{c}=200 \mathrm{~km}$. This profile has been constructed such that the effect of temperature relaxation becomes negligibly small beyond a distance of $L_{c}$ 
from $x=y=0$. The volume of shelf water that is subject to relaxation is indicated in Fig. 1. This parameterization is an idealized representation of localized dense water formation around Antarctica (Gordon 2009), and we emphasize that our goal is to analyze dynamical processes that control the export of dense water from the shelf, rather than those responsible for its formation. In our simulations dense water spreads rapidly along the continental shelf, so zonally uniform cooling over a longer time scale $T_{c}$ may be expected to produce similar results (see appendix).

We force the model ocean surface using zonally symmetric profiles of heat flux and zonal wind that approximate observations of the Southern Ocean (Large and Yeager 2009). These profiles are plotted in Fig. 1. The upward surface heat flux is given by

$$
Q(y)=Q_{0}\left\{2 \sin \left[\frac{2 \pi\left(y-L_{c}\right)}{L_{Q}}\right]-\sin \left[\frac{\pi\left(y-L_{c}\right)}{L_{Q}}\right]\right\},
$$

for $L_{c} \leq y \leq L_{c}+L_{Q}$, where $Q_{0}=10 \mathrm{~W} \mathrm{~m}^{-2}$ and $L_{Q}=$ $1700 \mathrm{~km}$. The surface is not thermally forced in the region of dense water formation, $0 \leq y \leq L_{c}$, and in the sponge layer at the northern boundary, described below. Our use of a fixed surface heat flux, rather than restoring the surface temperature, may somewhat reduce the sensitivity of the MOC to changes in the wind stress (Abernathey et al. 2011).

We choose our zonal wind profile such that the wind strengths over the ACC $\left(\tau_{\mathrm{ACC}}\right)$ and $\mathrm{ASF}\left(\tau_{\mathrm{ASF}}\right)$ may be varied independently without forming discontinuities in the wind stress, $\tau(y)$, or its curl, $-d \tau / d y$. Specifically, we prescribe

$$
\tau(y)= \begin{cases}\tau_{\mathrm{ACC}} \sin ^{2}\left[\frac{\pi\left(y-Y_{t}\right)}{L_{y}-Y_{t}}\right], & Y_{t} \leq y \leq L_{y}, \\ \tau_{\mathrm{ASF}} \sin ^{2}\left[\frac{\pi\left(Y_{t}-y\right)}{L_{\mathrm{ASF}}}\right], & Y_{t}-L_{\mathrm{ASF}} \leq y \leq Y_{t},\end{cases}
$$

and $\tau=0$ over $0 \leq y \leq Y_{t}-L_{\mathrm{ASF}}$. In our reference simulation, the transition between the ACC and ASF winds lies at $y=Y_{t}=800 \mathrm{~km}$, and the breadth of the ASF wind stress is $L_{\mathrm{ASF}}=600 \mathrm{~km}$. We achieve a surface mixed layer with a typical depth of $\sim 50 \mathrm{~m}$ using the $K$-profile parameterization (KPP) for vertical mixing (Large et al. 1994). KPP also restratifies statically unstable density configurations via convective adjustment using a temporarily large vertical diffusivity.

At the northern boundary we employ a "sponge layer" of width $100 \mathrm{~km}$ that parameterizes the diabatic processes required to close the overturning circulation in the northern ocean basins (Nikurashin and Vallis 2011; Wolfe and Cessi 2011). We relax the temperature toward an exponential profile motivated by Abernathey et al. (2011),

$$
\theta_{n}(z)=\theta_{0} \frac{\exp \left(z / H_{\theta}\right)-\exp \left(-H / H_{\theta}\right)}{1-\exp \left(-H / H_{\theta}\right)}
$$

which ranges from $0^{\circ} \mathrm{C}$ at $z=-H$ to $6^{\circ} \mathrm{C}$ at $z=0$ with a temperature scale height of $H_{\theta}=1000 \mathrm{~m}$. The temperature is relaxed most rapidly at $y=2000 \mathrm{~km}$, where the time scale is 7 days, and the relaxation rate decreases linearly to zero at $y=1900 \mathrm{~km}$. The relaxation profile (5) is close to the temperature at the northern boundary in simulations that do not employ a sponge layer. Our results are largely insensitive to changes in the prescribed temperature at the ocean bed, particularly over the continental shelf and slope. However, if a very cold $\left(\leq-0.5^{\circ} \mathrm{C}\right)$ relaxation temperature is prescribed then $\mathrm{AABW}$ exported from the shelf may no longer be dense enough to sink beneath the coldest waters at the northern boundary.

Our horizontal grid spacing of approximately $5 \mathrm{~km}$ is much smaller than the first Rossby radius of deformation in the ACC, which is around $20 \mathrm{~km}$. We therefore resolve the range of length scales that are unstable to baroclinic instability (Vallis 2006), which is the primary mechanism of eddy generation in the model. On the continental shelf the initial deformation radius may be as small as $5 \mathrm{~km}$, but experiments with grid spacings as small as $1 \mathrm{~km}$ do not lead to any substantial change in the eddy heat transport on the continental shelf.

The zonal wind stress [(4)] drives a strong eastward jet in the north and a weaker westward jet over the slope that are qualitatively similar to the ACC and ASF, respectively (Nowlin and Klinck 1986; Muench and Gordon 1995). We visualize this in Fig. 2 using the timeand zonal-mean zonal velocity $\bar{u}$. Throughout this article we denote zonal and time averages as $\bar{\bullet}^{x} \equiv L_{x}^{-1} \oint \bullet d x$ and $\boldsymbol{-}^{t} \equiv T^{-1} \int_{t_{0}}^{t_{0}+T} \bullet d t$, respectively, where $T=30 \mathrm{yr}$ unless otherwise stated, and $t_{0}$ is any time at which the simulation's total kinetic energy has reached a statistically steady state. We employ an unlabeled overbar as a shorthand for a zonal and time average $\overline{\boldsymbol{}} \equiv \overline{\boldsymbol{\bullet}}^{x}$, which we will refer to as the "mean" for convenience. The mean zonal velocity $\bar{u}$ is in thermal-wind balance, to a very good approximation, with the mean temperature, which is shown in Fig. 3a. In particular, our model captures the steeply sloping V-shaped isopycnals over the continental shelf break (Thompson and Heywood 2008).

The real ocean bed varies over a wide range of horizontal and vertical length scales and extracts momentum 


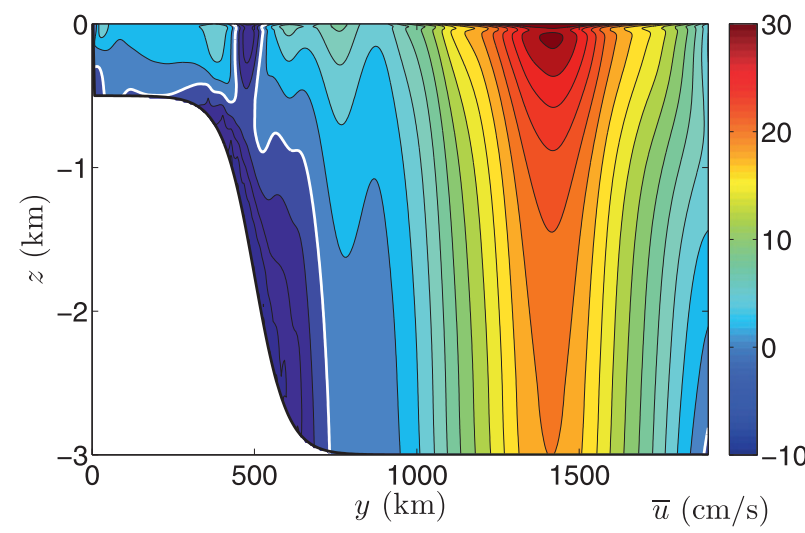

FIG. 2. Time- and zonal-mean zonal velocity $\bar{u}$ of our reference simulation in statistically steady state. The contour interval is $2 \mathrm{~cm} \mathrm{~s}^{-1}$ and the $\bar{u}=0$ contour is plotted in white.

from the ACC via form drag (Ferreira et al. 2005). In our simulations this is achieved via a linear bottom friction $r_{b}=1.1 \times 10^{-3} \mathrm{~m} \mathrm{~s}^{-1}$, so the mean basal velocity reaches $20 \mathrm{~cm} \mathrm{~s}^{-1}$ in the center of the ACC. This leads to a very large barotropic zonal transport of $\sim 330 \mathrm{~Sv}(1 \mathrm{~Sv} \equiv$ $10^{6} \mathrm{~m}^{3} \mathrm{~s}^{-1}$ ) between $y=800 \mathrm{~km}$ and $y=2000 \mathrm{~km}$, defined relative to a reference level at $z=-H$. However, the corresponding baroclinic transport of $80 \mathrm{~m}^{2} \mathrm{~s}^{-1}$ is much closer to $\sim 130 \mathrm{~m}^{2} \mathrm{~s}^{-1}$ transport of the real ACC (Nowlin and Klinck 1986). By contrast the total zonal transport beneath the polar easterlies (between $y=200 \mathrm{~km}$ and $y=$ $800 \mathrm{~km})$ is approximately $-0.7 \mathrm{~m}^{2} \mathrm{~s}^{-1}$ because the narrow westward ASF jet is abutted on both sides by strong eastward surface currents. We explain this structure dynamically using RMT in section 5 .

\section{b. Overturning circulation}

We diagnose the MOC using the meridional transport within isopycnal layers, defining the overturning

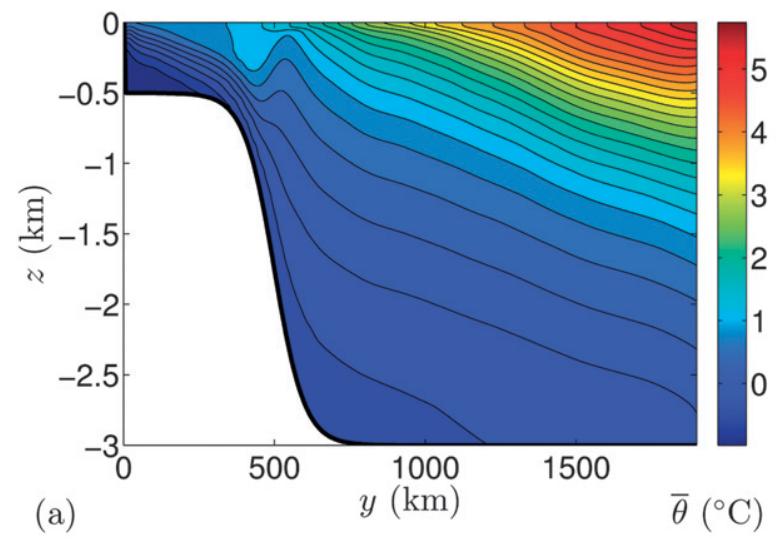

streamfunction $\psi$ as (Döös and Webb 1994; Abernathey et al. 2011)

$$
\psi(y, \theta)=\overline{\int_{\theta^{\prime}=\theta}^{z^{\prime}=0} v d z^{\prime}},
$$

where $z^{\prime}$ and $\theta^{\prime}$ are variables of integration. Figure $3 \mathrm{~b}$ shows $\psi$ mapped back to $y / z$ coordinates approximately using $\psi^{\dagger}(y, z)=\psi[y, \bar{\theta}(y, z)]$ (McIntosh and McDougall 1996). The MOC forms two superposed overturning cells: an upper (clockwise) cell and a lower (counterclockwise) cell. If the plotted overturning cells extended around a $\sim 17000-\mathrm{km}$ latitude band at $65^{\circ} \mathrm{S}$, the cells would each have a net transport of around $10 \mathrm{~Sv}$, comparable to observations (Lumpkin and Speer 2007).

The MOC is closely aligned with the mean isotherms in the ocean interior, where diapycnal mixing is weak. Strong diapycnal mixing and surface heat fluxes allow the MOC to cross mean isotherms rapidly in the mixed layer, as does the imposed temperature relaxation on the continental shelf and at the northern boundary. The streamlines of $\psi$ must also cross isopycnals along the ocean bed because this is the pathway of dense water exported from the continental shelf. Although the basal streamlines cross a much smaller range of temperatures than those in the surface mixed layer, this transformation of water masses nevertheless requires a large diapycnal diffusivity (Ito and Marshall 2008).

\section{c. Export of $A A B W$}

In Fig. 4a we present a Hovmöller diagram of the depth-averaged temperature $\bar{\theta}^{z}=h^{-1} \int_{-h}^{0} \theta d z$ in our reference simulation at $y=300 \mathrm{~km}$. The ubiquitous streaks of relatively warm and cold water correspond to eddies crossing this line of latitude southward and northward, respectively. Zhang et al. (2011) found similar patterns

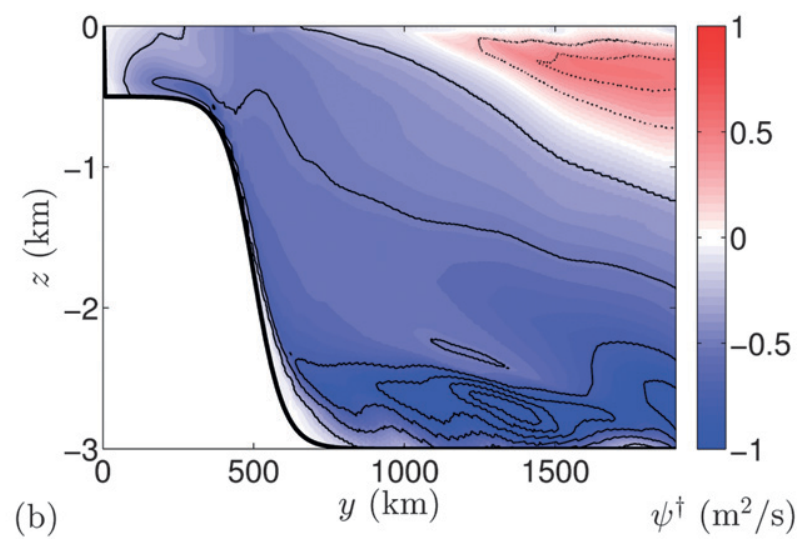

FIG. 3. (a) Mean stratification and (b) residual overturning streamfunction for our reference simulation. In (b) the dotted and solid lines highlight positive and negative streamlines respectively, with a contour interval of $0.25 \mathrm{~m}^{2} \mathrm{~s}^{-1}$. An overturning of $1 \mathrm{~m}^{2} \mathrm{~s}^{-1}$ corresponds to 1 Sv over the $1000-\mathrm{km}$ channel length. 

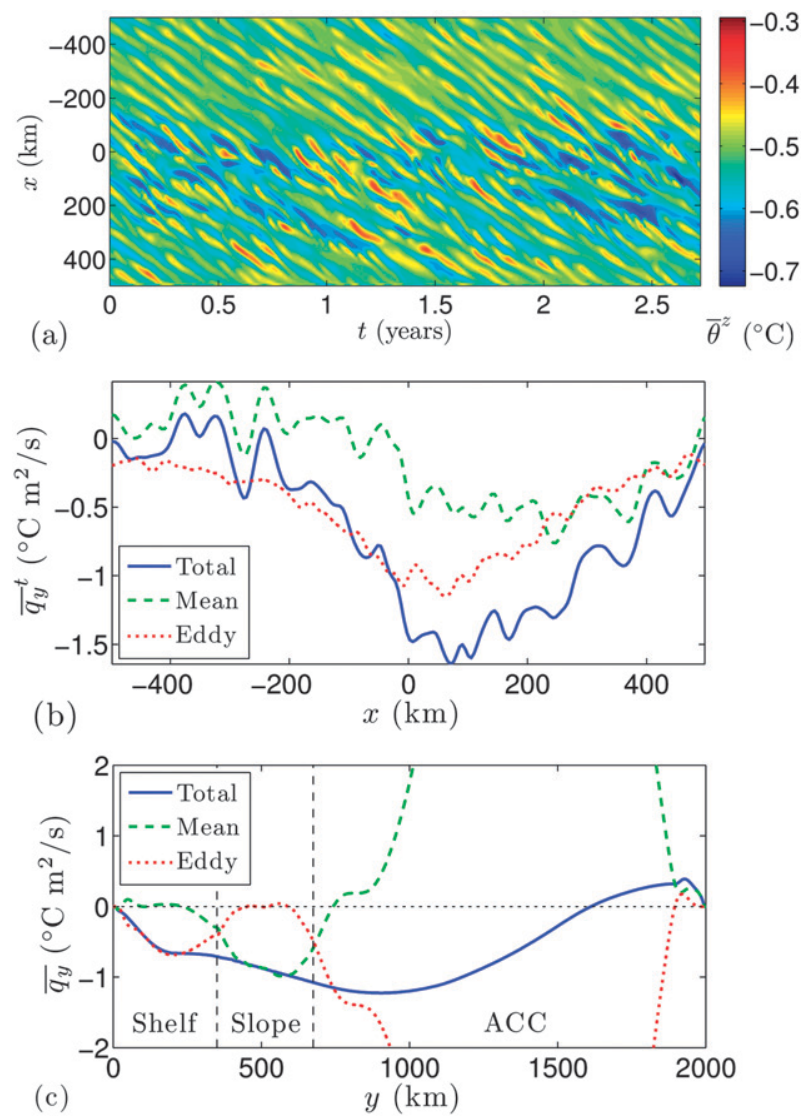

FIG. 4. (a) A Hovmöller diagram of the depth-averaged temperature in our reference simulation at $y=300 \mathrm{~km}$ is shown. Note the change in color scale from Fig. 1. (b) The time-mean meridional heat flux ${\overline{q_{y}}}^{t}$ across $y=300 \mathrm{~km}$ is shown. (c) The time- and zonalmean heat flux $\overline{q_{y}}$ as a function of latitude is shown.

of tracer export from a bay on the continental shelf in an idealized two-layer configuration. Export of the coldest water is concentrated between $x \approx-100 \mathrm{~km}$ and $x \approx 250 \mathrm{~km}$, centered around $75 \mathrm{~km}$ eastward of the most rapid bottom water formation at $x=0$. This is due to advection of eddies by the mean zonal velocity, which is around $2 \mathrm{~cm} \mathrm{~s}^{-1}$ at this latitude and is visualized by the diagonal streaks in Fig. 4a.

We quantify the eddy heat transport in Fig. 4b by defining the vertically integrated meridional heat flux

$$
{\overline{q_{y}}}^{t}=\int_{z=-h}^{z=0} d z \overline{v \theta}^{t}=\int_{z=-h}^{z=0} d z\left(\bar{v}^{t} \bar{\theta}^{t}+{\overline{v^{\prime} \theta^{t}}}^{t}\right)
$$

which we have decomposed into mean and "eddy" components. Here primes denote deviations from the time mean, for example, $\theta^{\prime}=\theta-\bar{\theta}^{t}$. The time average is taken over 130 years in statistically steady state to minimize the influence of topographic Rossby waves whose propagation speeds are very close to that of the slope

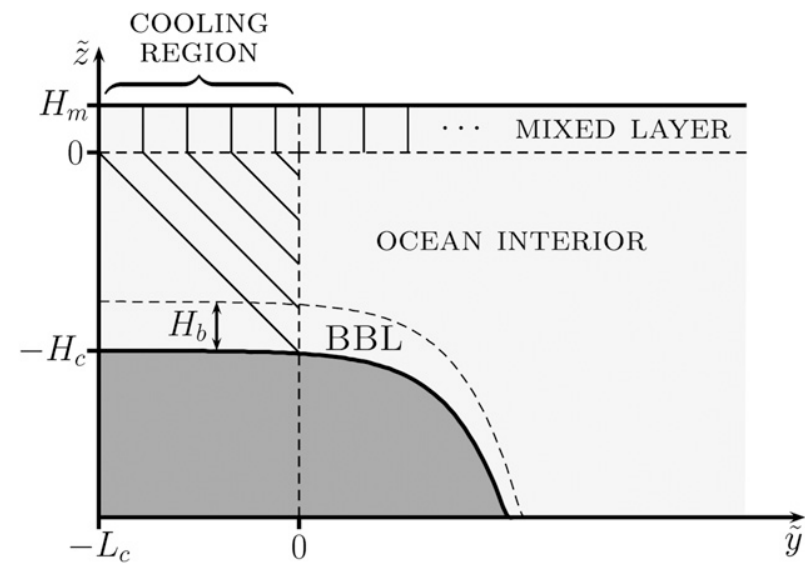

FIG. 5. Schematic of the continental shelf. Dashed lines illustrate the conceptual division of the domain into a mixed layer, a cooling region, a bottom boundary layer (BBL), and the interior. Thin solid lines indicate the assumed temperature stratification in the mixed layer and in the shelf cooling region. Note that $\tilde{y}=y-L_{c}$ and $\tilde{z}=z+H_{m}$.

current. The imposed cooling on the shelf leads to a patch of denser water forming in the region $x^{2}+y^{2} \leq L_{c}$, visible in Fig. 1. This gives rise to a mean eastward baroclinic flow, via thermal wind balance, that transports water off the shelf in $x<0$ and onto the shelf in $x>0$. This accounts for the zonal asymmetry in the mean component of ${\overline{q_{y}}}^{t}$ in Fig. 4b. The eddy component of ${\overline{q_{y}}}^{t}$ is everywhere negative and corresponds to a southward heat transport that is most intense at $x \approx 75 \mathrm{~km}$, as suggested by Fig. 4a.

In Fig. $4 \mathrm{c}$ we plot the time- and zonal-mean meridional heat flux $\overline{q_{y}}$ as a function of latitude. The northern surface heating and southern surface/shelf cooling lead to a southward heat transport over most of the domain. In the ACC, the net heat flux is small compared to the large northward mean and southward eddy heat fluxes. On the continental shelf the heat transport is dominated by eddies, transporting cold water northward to the shelf break. On the continental slope the eddy heat transport vanishes, and downslope flow takes place in a frictional bottom boundary layer. The mean down-slope velocity between $y=400 \mathrm{~km}$ and $y=600 \mathrm{~km}$ is $0.9 \mathrm{~cm} \mathrm{~s}^{-1}$, and the mean along-slope velocity is $-0.63 \mathrm{~cm} \mathrm{~s}^{-1}$. Outflowing dense water takes around 403 days to traverse the slope, during which it travels around $2300 \mathrm{~km}$ westward.

\section{Residual-mean theory}

To develop a theory of our model overturning circulation, we conceptually decompose the model domain into a surface mixed layer of depth $H_{m}$, a shelf cooling region of meridional extent $L_{c}$, a bottom boundary layer 
of thickness $H_{b}$, and the ocean interior. Figure 5 illustrates this decomposition and the assumed temperature stratification in the surface mixed layer and shelf cooling region. For convenience we define a translated system of Cartesian coordinates $(\tilde{y}, \tilde{z})=\left(y-L_{c}, z+H_{m}\right)$, in which $(0,0)$ lies at the upper southern corner of the ocean interior.

\section{a. Residual-mean equations}

We derive the core equations of residual-mean theory following Marshall and Radko (2003, 2006). Averaging the material conservation equation for temperature, we obtain

$$
J\left(\psi^{\dagger}, \bar{\theta}\right)=S_{\theta}+\nabla \cdot(\kappa \nabla \bar{\theta}) \text { and } \psi^{\dagger}=\bar{\psi}+\psi^{\star},
$$

where $\nabla \equiv\left(\partial_{\tilde{y}}, \partial_{\tilde{z}}\right)$ is the two-dimensional gradient operator. The mean temperature $\bar{\theta}$ is advected by the residual streamfunction, subject to mean temperature sources/sinks $S_{\theta}$ and mixing $\kappa$ across temperature surfaces. Following Marshall and Radko (2003), we relate the mean streamfunction $\bar{\psi}$ in the ocean interior to the surface wind stress,

$$
\bar{\psi}=-\frac{\tau(\tilde{y})}{\rho_{0} f_{0}},
$$

which is valid in our zonally periodic domain in the limit of vanishingly small Rossby number and viscosity. We have also approximated $f$ by its reference value $f_{0}$.

The eddy streamfunction $\psi^{\star}$ and diapycnal diffusivity $\kappa$ are defined as the components of the advective eddy heat flux directed along and across mean isotherms, respectively (Plumb and Ferrari 2005),

$$
\psi^{\star}=\frac{\overline{\boldsymbol{v}^{\prime} \theta^{\prime}} \times \nabla \bar{\theta}}{|\nabla \bar{\theta}|^{2}} \quad \text { and } \quad \kappa=-\frac{\overline{\boldsymbol{v}^{\prime} \theta^{\prime}} \cdot \nabla \bar{\theta}}{|\nabla \bar{\theta}|^{2}} .
$$

To close (8) we parameterize the eddy heat flux $\overline{\boldsymbol{v}^{\prime} \theta^{\prime}}$ following Gent and McWilliams (1990), assuming that eddies transport heat down the meridional temperature gradient,

$$
\overline{v^{\prime} \theta^{\prime}}=-K \frac{\partial \bar{\theta}}{\partial \tilde{y}}, \Rightarrow \psi^{\star}=(K-\kappa) s_{\theta} .
$$

Here $K>0$ is the isopycnal or eddy diffusivity and $s_{\theta}=-\partial_{\tilde{y}} \bar{\theta} / \partial_{\tilde{z}} \bar{\theta}$ is the isotherm slope. This definition of $\psi^{\star}$ differs from that used by Marshall and Radko (2003) by a term proportional to $\kappa$. We will later neglect this term under the assumption of small aspect ratio.

A basic constraint on the streamfunctions $\bar{\psi}$ and $\psi^{\star}$ is that the instantaneous mass and heat transports through solid boundaries should be zero. In our zonally symmetric domain this constraint may be satisfied by imposing

$$
\bar{\psi}=0, \quad \psi^{\star}=0 \quad \text { and } \quad \nabla \bar{\theta} \cdot \mathbf{n}=0 \quad \text { on } \quad \partial S,
$$

where $\partial S$ denotes the domain boundary and $\mathbf{n}$ denotes the unit normal vector. In the appendix we employ (12) to derive boundary conditions for the surface mixed layer, the shelf cooling region, the ocean bed, and the northern relaxation region.

\section{b. Nondimensionalization}

To simplify our analysis of the residual-mean (8), we nondimensionalize using the following scales (Nikurashin and Vallis 2011):

$$
\begin{gathered}
\psi^{\dagger}, \psi^{\star}, \bar{\psi} \sim \frac{\tau_{0}}{\rho_{0}\left|f_{0}\right|}, \quad \tilde{y} \sim \tilde{L}_{y}, \quad \tilde{z} \sim \tilde{H}, \quad \tau \sim \tau_{0}, \quad \bar{\theta} \sim \theta_{0}, \\
\Theta_{m} \sim \frac{\theta_{0} \tau_{0}}{\rho_{0}\left|f_{0}\right| \tilde{L}_{y}}, \quad V_{c} \sim \frac{\tau_{0}}{\rho_{0}\left|f_{0}\right| \tilde{H}}, \quad \kappa=\kappa_{0} k, \\
K=\frac{\tilde{L}_{y} \tau_{0}}{\tilde{H} \rho_{0}\left|f_{0}\right|} \Lambda, \quad \varepsilon=\frac{\kappa_{0} \tilde{L}_{y} \rho_{0}\left|f_{0}\right|}{\tilde{H} \tau_{0}}, \quad \text { and } \quad \mu=\frac{\tilde{H}}{\tilde{L}_{y}} .
\end{gathered}
$$

Here $\tilde{H}=H-H_{m}, \kappa_{0}>0, \tau_{0}>0$, and $f_{0}<0$ are scales for the depth, diapycnal diffusivity, wind stress and Coriolis parameter, respectively. The downward surface heat flux $\Theta_{m}$ and shelf piston velocity $V_{c}$ are defined in the appendix. We denote dimensionless variables using a hat. The dimensionless isopycnal and diapycnal diffusivities, $\Lambda$ and $k$, may vary arbitrarily and will be discussed in section 4 . For typical parameter values of $K=2000 \mathrm{~m}^{2} \mathrm{~s}^{-1}, \kappa_{0}=1 \times 10^{-4} \mathrm{~m}^{2} \mathrm{~s}^{-1}, \tau_{0}=0.2 \mathrm{~N} \mathrm{~m}^{-2}$, and $f_{0}=1 \times 10^{-4} \mathrm{~s}^{-1}$, we obtain $\Lambda \approx 1.7, \varepsilon=3 \times 10^{-2}$, and $\mu \approx 1.7 \times 10^{-3}$. Neglecting terms of $\mathcal{O}\left(\mu^{2}\right)$ throughout, the dimensionless residual-mean equations and boundary conditions may be summarized as

$$
J\left(\hat{\psi}^{\dagger}, \hat{\theta}\right)=\varepsilon \frac{\partial}{\partial \hat{z}}\left(k \frac{\partial \bar{\theta}}{\partial \hat{z}}\right)
$$

$$
\hat{\psi}^{\dagger}=\hat{\bar{\psi}}+\hat{\psi}^{\star}, \quad \hat{\psi}^{\star}=\Lambda \hat{s}_{\theta} F_{b}(\hat{z}), \quad \hat{\bar{\psi}}=\hat{\tau} F_{b}(\hat{z}),
$$

$$
\begin{aligned}
\hat{\psi}^{\dagger}(\hat{y}, 0) & =\left[\frac{\partial \hat{\theta}}{\partial \hat{y}}(\hat{y}, 0)\right]^{-1} \hat{\Theta}_{m}(\hat{y}), \\
\hat{\psi}^{\dagger}(0, \hat{z}) & =\hat{V}_{c} \frac{\left[\hat{\theta}(0, \hat{z})-\hat{\theta}_{c}\right]}{\partial_{\hat{z}} \hat{\theta}(0, \hat{z})} I(\hat{z}), \\
\frac{\partial \hat{\theta}}{\partial \hat{z}}(0,-\hat{h}) & =0, \text { and } \\
\hat{\theta}\left(1-\hat{L}_{n}, \hat{z}\right) & =\hat{\theta}_{n}(\hat{z}) .
\end{aligned}
$$

The derivation of boundary conditions (14c)-(14f) is given in the appendix as are the definitions of the vertical structure functions $F_{b}(z)$ and $I(z)$. 


\section{Isopycnal and diapycnal diffusivities}

In this section we develop a parameterization for $K$ and $\kappa$ that is suitable for our model problem. Ideally our theory should not be conditioned by our simulations, so that their results may be fairly compared. However, our simulations exhibit dramatic changes in $K$ over the continental slope, and in $\kappa$ close to the ocean bed, which our theory must address to capture key features of the stratification and overturning circulation. We therefore motivate parameterizations of $K$ and $\kappa$ using our reference simulation, described in section 2 , and then test the theory against simulations with a range of different surface wind stress profiles in section 6 . In this section we return to dimensional variables for convenience.

\section{a. Eddy fluxes over the continental slope}

Recent studies using numerical simulations (Isachsen 2011) and laboratory experiments (Pennel et al. 2012) have shown that sloping topography may stabilize coastal currents because it suppresses baroclinic instability. Following Isachsen (2011) and Pennel et al. (2012), we quantify this effect using the topographic parameter $\delta$,

$$
\delta=\frac{s_{b}}{s_{\theta}},
$$

where $s_{b}=-d_{y} h$ and $s_{\theta}=-\partial_{y} \bar{\theta} / \partial_{z} \bar{\theta}$ as in section 3 . Isachsen (2011) constructed an eddy diffusivity using the growth rates of the Eady problem over a sloping bottom using the approach of Stone (1972),

$$
\left.K(\delta) \propto \max (\sigma) \cdot \lambda^{2}\right|_{\sigma=\max (\sigma)} .
$$

Here $\sigma$ is the growth rate, and $\lambda$ is the wavelength. In Fig. 6 we plot $K$ against $\delta$, as diagnosed from our reference simulation at all points in the $y / z$ plane via (11), excluding those in the surface mixed layer and in the lowest grid cells. We also plot the theoretical diffusivity (16) from the two-layer quasigeostrophic (QG) equations with equal layer depths (see e.g., Pavec et al. 2005; Pennel et al. 2012), rescaled to fit the simulation data approximately.

The diagnosed $K$ in Fig. 6 is qualitatively similar to the results of Isachsen (2011) for $\delta \geq 0$, but differs for $\delta<0$. In our simulations the baroclinic stability may be altered by the strong depth-dependence of the isopycnal slope $s_{\theta}$ in regions where $\delta<0$, in contrast to the experiments of Isachsen (2011). The QG theory predicts that $K$ should be zero for $\delta>1$, and that $K \rightarrow 0$ as $\delta \rightarrow-\infty$, whereas the diagnosed $K$ is nonzero as $\delta \rightarrow \pm \infty$. This results in the smooth temperature extrema over the continental shelf in Fig. 3a. However, it should be noted

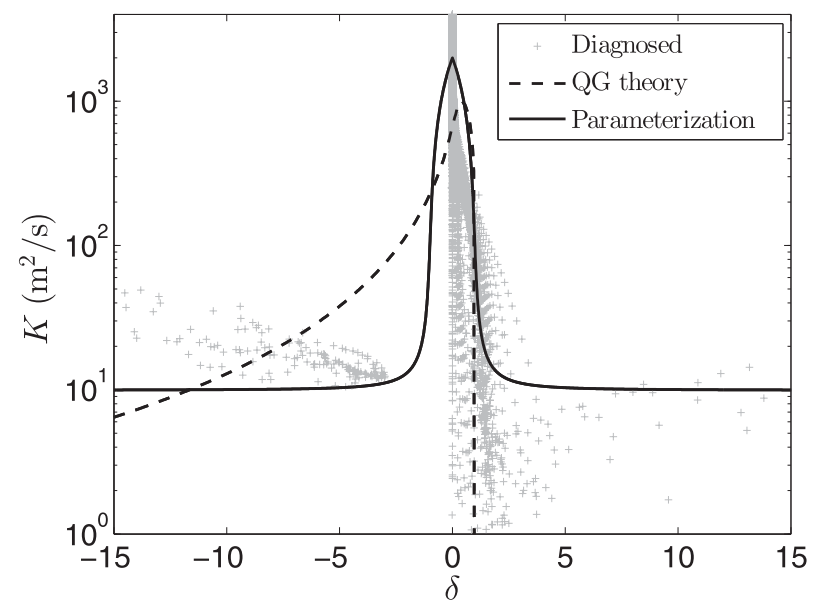

FIG. 6. Eddy diffusivity $K$ diagnosed via (11) from our reference simulation (points), constructed via (16) from two-layer baroclinic instability theory (dashed line), and as given by our parameterization (17) with $K_{0}=2000 \mathrm{~m}^{2} \mathrm{~s}^{-1}$ (solid line). There are few diagnosed values of $K$ between $\delta=-3$ and $\delta=0$ because in this simulation the isotherm slope is only positive where the continental slope is very steep.

that the variation in depth in our simulations lies well outside the range of validity of QG theory.

Further work is required to develop an accurate parameterization of eddy transports over the continental slope, but such an undertaking is beyond the scope of this paper. Instead, we parameterize heuristically the dramatic reduction in $K$ when $s_{b}$ becomes comparable to $s_{\theta}$. Our goal is to capture the key features of $K(\delta)$ in our reference simulation in order to develop a simple theory of the model's overturning and stratification. Specifically, we choose

$$
\begin{aligned}
K= & K_{0}\left[1+\frac{1}{2} \sqrt{(1-|\delta|)^{2}+4 \gamma^{2}|\delta|^{2}}\right. \\
& \left.-\frac{1}{2} \sqrt{(1+|\delta|)^{2}+4 \gamma^{2}|\delta|^{2}}\right]
\end{aligned}
$$

where $\gamma \ll 1$ and $K_{0}$ is constant. When $\gamma=0$, (17) simplifies to $K=K_{0}(1-|\delta|) \mathcal{H}(1-|\delta|)$, where $\mathcal{H}$ denotes the Heaviside function. In this case $K=0$ for $|\delta|>1$, similar to the QG theory when $\delta>0$. For nonzero $\gamma,(17)$ has the property that $K=\gamma K_{0}+\mathcal{O}\left(\gamma^{2}\right)$ for $\delta=1$, so $K$ is reduced by a factor of $\gamma$ when the isopycnal and topographic slopes have the same magnitude. In the limit $\delta \rightarrow \pm \infty,(17)$ asymptotes to $K \rightarrow 2 \gamma^{2} K_{0}$. In Fig. 6 we plot (17) for the case $\gamma=0.05$; we use this value in all solutions presented in this paper.

\section{b. Deep diapycnal mixing}

In our simulations we prescribe a uniform vertical diffusivity of $\kappa_{v}=5 \times 10^{-6} \mathrm{~m}^{2} \mathrm{~s}^{-1}$, so cross-isopycnal 


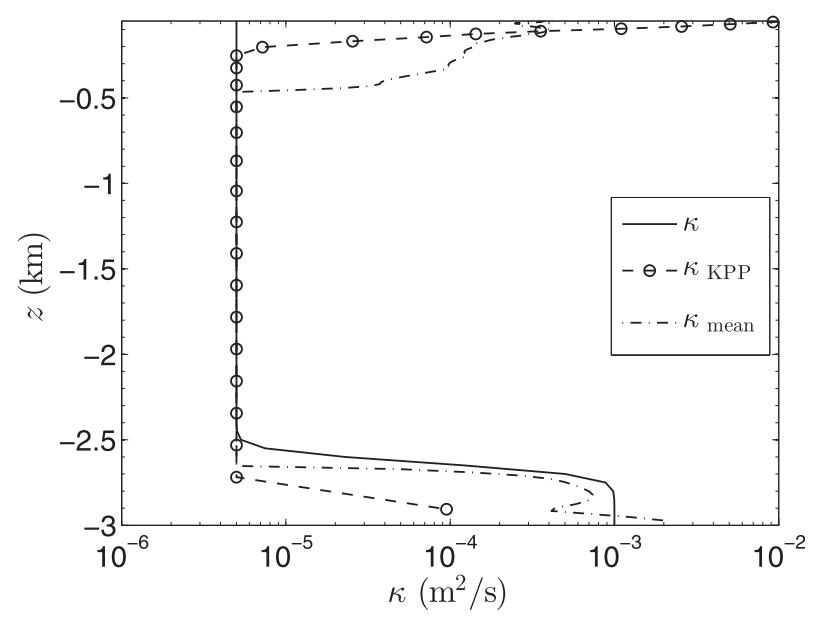

FIG. 7. Mean vertical diffusivity profiles supplied by KPP (points) and diagnosed directly from the heat fluxes in our reference simulation (dashed-dotted line), and our theoretical parameterization in (18) of $\kappa$ (solid line).

flow in the deep ocean is achieved via a combination of convective mixing and grid-scale numerical diffusion because of the flux-limited temperature advection scheme. In Fig. 7 we plot as a function of depth the mean diffusivity supplied by KPP $\left(\kappa_{\mathrm{KPP}}\right)$, where the mean has been taken in $x, t$, and over $y \in(800 \mathrm{~km}, 1900 \mathrm{~km})$. For comparison, we also plot the diffusivity diagnosed directly from the cross-isotherm component of the mean meridional and vertical eddy heat fluxes $\left(\kappa_{\text {mean }}\right)$ via $(10)$.

The rapid increase in $\kappa$ close to the ocean bed results in a diffusive heat sink on the right-hand side of (14a), which must be balanced by residual advection down the mean temperature gradient. This gives rise to the intense $\left(\sim 1.7 \mathrm{~m}^{2} \mathrm{~s}^{-1}\right)$ recirculating overturning cell visible at the bottom of Fig. 3b. The magnitude of $\kappa_{\text {mean }}$, and therefore of the deep recirculation, may be reduced by increasing the vertical resolution and employing an alternative temperature advection scheme (see Hill et al. 2012). However, these modifications change $\psi$ by at most $1 \%-2 \%$ everywhere else in the domain, particularly over the continental shelf and slope.

The diagnosed diffusivity profiles $\kappa_{\mathrm{KPP}}$ and $\kappa_{\text {mean }}$ motivate the following choice of $\kappa$ for RMT,

$$
\kappa(y, z)=\kappa_{0}+\frac{1}{2}\left(\kappa_{b}-\kappa_{0}\right)\left[1-\tanh \left(\frac{z+h-Z_{\kappa}}{H_{\kappa}}\right)\right],
$$

where $\kappa_{0}=5 \times 10^{-6} \mathrm{~m}^{2} \mathrm{~s}^{-1}, \kappa_{b}=1 \times 10^{3} \mathrm{~m}^{2} \mathrm{~s}^{-1}, Z_{k}=$ $250 \mathrm{~m}$, and $H_{k}=50 \mathrm{~m}$. The diffusivity profile in the ACC is shown in Fig. 7. This parameterized deep diapycnal mixing may be viewed as an artifice of our numerical configuration. However, strong diapycnal mixing is required for bottom water to cross isopycnals close to the ocean bed (Ito and Marshall 2008), and $\kappa$ has been observed to increase by two or more orders of magnitude in parts of the Southern Ocean (Garabato et al. 2004). Furthermore, our residual-mean solutions in section 5 develop unrealistically strong stratification unless $\kappa$ is comparable to the profile shown in Fig. 7.

\section{Solution of the residual-mean equations}

\section{a. Numerical solutions in terrain-following coordinates}

We create an iterative scheme to solve (14a)-(14f) by introducing an artificial time derivative into (14a), following Nikurashin and Vallis (2011, 2012),

$$
\frac{\partial \hat{\theta}}{\partial \hat{t}}+J\left(\hat{\psi}^{\dagger}, \hat{\theta}\right)=\varepsilon \frac{\partial}{\partial \hat{z}}\left(k \frac{\partial \hat{\theta}}{\partial \hat{z}}\right) .
$$

Here the scale for time $t$ is $\rho_{0}\left|f_{0}\right| L \tilde{H} / \tau_{0} \sim 80 \mathrm{yr}$. We rewrite $\hat{\theta}$ as a function of $y$ and $\zeta$, where $\zeta=(z / h) H$ is a terrain-following vertical coordinate, then discretize (19) on an Arakawa C grid and apply the finite-volume scheme of Kurganov and Tadmor (2000). We integrate forward in time using the total variation diminishing Runge-Kutta scheme of Shu and Osher (1989) until the computation reaches a steady state.

In Fig. 8 we plot the stratification and overturning circulation computed using parameters appropriate to our reference simulation, with $K_{0}=2000 \mathrm{~m}^{2} \mathrm{~s}^{-1}$. This solution, and all of those described below in section 6 , has been computed on a numerical grid of $150 \times 150$ points. Our theory captures the qualitative features of the stratification and overturning circulation throughout the domain. The region of anomalously weak overturning adjacent to the continental slope is due to our simplified parameterization in (18) of deep diapycnal mixing. This region may be corrected using a more sophisticated parameterization that permits latitudinal variation of $\kappa_{b}, H_{\kappa}$, and $Z_{\kappa}$, but it has little impact on our results. Both our theory and our eddy-resolving simulations predict upper and lower overturning cell strengths of around $0.6 \mathrm{~m}^{2} \mathrm{~s}^{-1}$ and $0.5 \mathrm{~m}^{2} \mathrm{~s}^{-1}$. In section 6 we quantify the predictive skill of our theory over a range of surface wind stress profiles.

\section{b. Structure of the Antarctic Slope Front}

In the limit of vanishingly small diapycnal mixing $(\varepsilon \rightarrow 0)$, the residual-mean temperature (14a) may be solved asymptotically along individual isotherms. We rewrite (14a)-(14f) in $\hat{y} / \hat{\theta}$ coordinates via the transformation $\hat{z}=\hat{z}(\hat{y}, \hat{\theta}), \hat{\psi}^{\dagger}=\hat{\psi}^{\dagger}[\hat{y}, \hat{z}(\hat{y}, \hat{\theta})]$, under which the isotherm slope $\hat{s}_{\theta}$ may be written as 


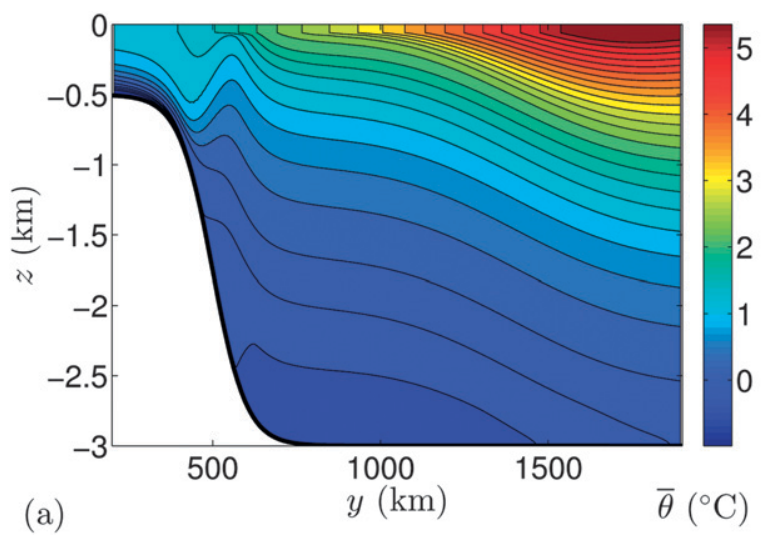

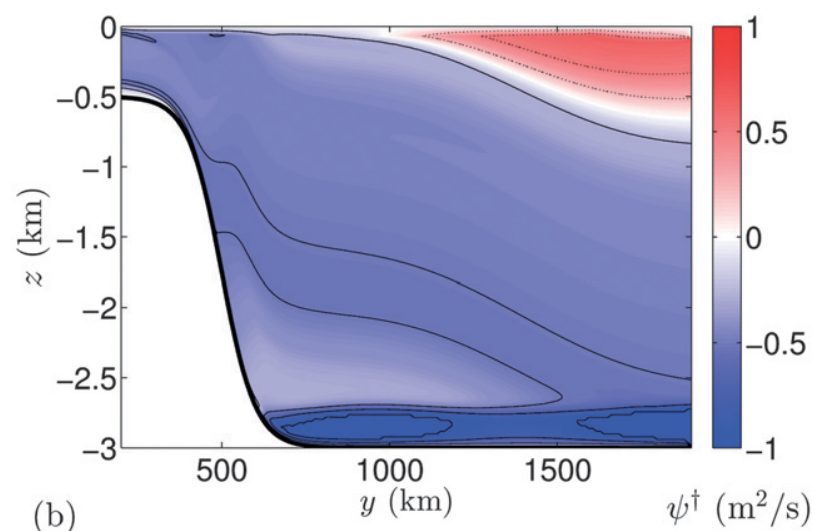

(b)

FIG. 8. As in Fig. 3, but with $\bar{\theta}$ and $\psi^{\dagger}$ calculated numerically via RMT using (19). Units are dimensional.

$$
\left.\frac{\partial \hat{z}}{\partial \hat{y}}\right|_{\hat{\theta}}=\hat{s}_{\theta}=-\frac{\partial_{\hat{y}} \hat{\theta}}{\partial_{\hat{z}} \hat{\theta}} .
$$

We will integrate this equation to determine the shapes of isotherms that originate from the shelf cooling region at $\hat{y}=0$. The isotherm slope $\hat{s}_{\theta}$ is determined via (14b) as

$$
\hat{s}_{\theta} \Lambda\left(\hat{s}_{\theta}\right)=\hat{\psi}^{\dagger}(\hat{y}, \hat{\theta})-\hat{\tau}(\hat{y})
$$

Here we assume for simplicity that the isotherm enters neither the bottom boundary layer nor the surface mixed layer, so $F_{b}(\hat{z}) \equiv 1$. To determine the change in $\hat{\psi}^{\dagger}$ along the isotherm, we use (20) to write the Jacobian $J\left(\hat{\psi}^{\dagger}, \hat{\theta}\right)$ as

$$
J\left(\hat{\psi}^{\dagger}, \hat{\theta}\right)=\frac{\partial \hat{\theta}}{\partial \hat{z}}\left(\left.\frac{\partial \hat{\psi}^{\dagger}}{\partial \hat{y}}\right|_{\hat{z}}+\left.\hat{s}_{\theta} \frac{\partial \hat{\psi}^{\dagger}}{\partial \hat{z}}\right|_{\hat{y}}\right)=\left.\frac{\partial \hat{\theta}}{\partial \hat{z}} \frac{\partial \hat{\psi}^{\dagger}}{\partial \hat{y}}\right|_{\hat{\theta}},
$$

where the rightmost equality follows from an application of the chain rule. Substituting (22) into (14a) yields an equation for the rate of change of $\hat{\psi}^{\dagger}$ along isotherms, which we solve via an asymptotic expansion in $\varepsilon$,

$$
\left.\frac{\partial \hat{\psi}^{\dagger}}{\partial \hat{y}}\right|_{\hat{\theta}}=\mathcal{O}(\varepsilon) \Rightarrow \hat{\psi}^{\dagger}=\hat{\psi}_{0}^{\dagger}(\hat{\theta})+\mathcal{O}(\varepsilon)
$$

where $\hat{\psi}_{0}^{\dagger}(\hat{\theta})=\hat{\psi}^{\dagger}[0, \hat{z}(0, \hat{\theta})] \leq 0$ by the shelf boundary condition $(14 \mathrm{~d})$.

As $\Lambda>0$ for all $\delta$, it follows from (21) that the sign of the isotherm slope is determined by the relative strengths of the mean and residual streamfunctions, or $\operatorname{sign}\left(\hat{s}_{\theta}\right)=\operatorname{sign}\left(\hat{\psi}_{0}^{\dagger}-\hat{\tau}\right)$ to leading order in $\varepsilon$. If the residual streamfunction is stronger than the wind-driven overturning on the slope, $\hat{\psi}_{0}^{\dagger}(\hat{\theta})<\hat{\tau}_{\mathrm{ACC}}=\min _{y}(\hat{\tau})$, then $\hat{s}_{\theta}<0$ for all $y$, and so the isotherm does not form a
V shape. This accurately describes the coldest isotherms emanating from the shelf in Figs. 3a and 8a. If the residual streamfunction is weaker than the wind-driven overturning on the slope, $\hat{\tau}_{\mathrm{ACC}}<\hat{\psi}_{0}^{\dagger}<0$, then the form of the surface wind stress (4) means that $\hat{s}_{\theta}$ changes sign twice between $\hat{y}=0$ and $\hat{y}=\hat{L}_{\mathrm{ASF}}$. This presents as a $\mathrm{V}$-shaped ASF in Figs. 3a and 8a. The sharpness of the $\mathrm{V}$ is accentuated because of the reduction in $\Lambda$ by a factor of around 40 over the continental slope, due to suppression of baroclinic instability (Isachsen 2011).

To emphasize the role of eddy suppression in shaping the ASF, in Fig. 9a we plot the residual-mean stratification produced by a uniform isopycnal diffusivity. The shelf and slope stratification no longer resembles the structure diagnosed from our reference simulation (see Fig. 3), and the warmer shelf water doubles the strength of the deep overturning circulation (not shown). If there is no bottom water formation on the shelf $\left[\hat{\psi}^{\dagger}(0, z) \equiv 0\right]$ then (21) yields $\hat{s}_{\theta}>0$. In this case the isotherms originating from the northern boundary would simply plunge into the continental slope, as observed in the Eastern Weddell Sea (Chavanne et al. 2010) and other parts of the Antarctic margins where the continental shelf is narrow (Whitworth et al. 1998). This configuration is illustrated in Fig. 9b, which shows the residual-mean stratification produced by setting the surface buoyancy forcing and shelf cooling to zero.

\section{c. A constraint on the deep overturning strength}

Even under an expansion in $\varepsilon$ the system (20)-(21) remains analytically intractable. We therefore pose an additional asymptotic expansion of our isopycnal diffusivity $\Lambda$ in the parameter $\gamma$,

$$
\Lambda\left(\hat{s}_{\theta}\right)=\Lambda_{0}(1-|\delta|) \mathcal{H}(1-|\delta|)+\mathcal{O}\left(\gamma^{2}\right),
$$

where $\delta=\hat{s}_{b} / \hat{s}_{\theta}$ as in section 4 . Substituting this expansion into (21) yields 

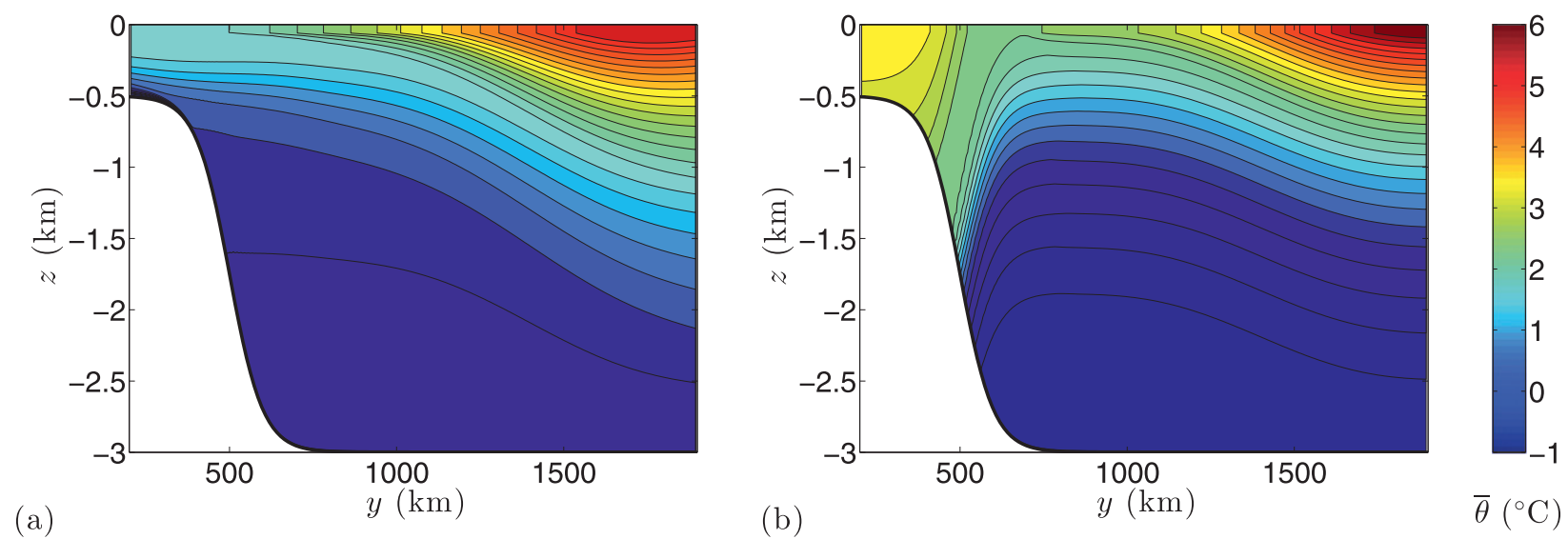

FIG. 9. Plots of the residual-mean temperature stratification, corresponding to our reference simulation with alternative parameter choices. In (a) we have assumed a uniform isopycnal diffusivity $K=2000 \mathrm{~m}^{2} \mathrm{~s}^{-1}$ everywhere, including over the continental slope. In (b) we have turned off the forcing on the continental shelf $\left(V_{c}=0\right.$, see the appendix) and at the ocean surface $\left(\Theta_{m}=0\right)$, so the residual overturning is vanishingly small.

$$
\hat{s}_{\theta}=\frac{\hat{\psi}_{0}^{\dagger}-\hat{\tau}}{\Lambda_{0}}+\operatorname{sign}\left(\hat{\psi}_{0}^{\dagger}-\hat{\tau}\right)\left|\hat{s}_{b}\right|+\mathcal{O}\left(\varepsilon, \gamma^{2}\right) .
$$

Substituting this approximation into (20), we may integrate exactly to obtain the change in isotherm height above the ocean bed between the southern and northern boundaries,

$$
\begin{aligned}
(\hat{z}+\hat{h})_{\hat{y}=0}^{\hat{y}=1}= & \frac{1}{\Lambda_{0}}\left(\hat{\psi}_{0}^{\dagger}-\frac{1}{2} \hat{\tau}_{\mathrm{ASF}} \hat{L}_{\mathrm{ASF}}-\frac{1}{2} \hat{\tau}_{\mathrm{ACC}} \hat{L}_{\mathrm{ACC}}\right) \\
& +2\left(1-\hat{H}_{s}\right) \tanh \left(\frac{\xi \hat{L}_{\mathrm{ASF}}}{2 \hat{W}_{s}}\right)+\mathcal{O}\left(\varepsilon, \gamma^{2}\right),
\end{aligned}
$$

where $\hat{H}_{s}=\tilde{H}_{s} / H$ is the dimensionless shelf depth excluding the surface mixed layer. Here we have ignored the relaxation region close to the northern boundary for simplicity, and the parameter $\xi$ is defined as

$$
\xi= \begin{cases}\frac{2}{\pi} \cos ^{-1}\left(\sqrt{\frac{\hat{\psi}_{0}^{\dagger}}{\hat{\tau}_{\mathrm{ASF}}}}\right), & \hat{\tau}_{\mathrm{ASF}} \leq \hat{\psi}_{0}^{\dagger}<0, \\ 0, & \hat{\psi}_{0}^{\dagger} \leq \hat{\tau}_{\mathrm{ASF}} .\end{cases}
$$

Because of the formation of dense water on the continental shelf, colder water exists at the southern boundary than at the northern boundary. In $\hat{y} / \hat{\theta}$ coordinates, any isotherm $\hat{z}(\hat{y}, \hat{\theta})$ that originates from these depths at the southern boundary $\left[\hat{z}(0, \hat{\theta})<\hat{z}_{0}\right]$ must enter the bottom boundary layer before it reaches $\hat{y}=1$, so we require that $\hat{z}(1, \hat{\theta})<-1$. A necessary condition is that the lefthand side of (26) must be less than zero. Therefore, the right-hand side of (26) must be less than zero, to leading order in $\varepsilon$ and $\gamma^{2}$. This serves to constrain the strength of the deep overturning circulation, measured by $\hat{\psi}_{0}^{\dagger}$. By setting the right-hand side of (26) to zero and solving for $\hat{\psi}_{0}^{\dagger}$, we predict the sensitivity of the deep overturning circulation to changes in the polar easterlies $\tau_{\mathrm{ASF}}$. In section 6 this prediction is compared with those of our numerical RMT solutions and our eddy-resolving simulations. The last term on the right-hand side of (26) typically dominates the others unless $\xi=0$, so an approximate constraint is that $\hat{\psi}_{0}^{\dagger}(\hat{\theta}) \lesssim \hat{\tau}_{\mathrm{ASF}}$, or in dimensionless variables,

$$
\max _{\tilde{y}=0}\left|\psi^{\dagger}\right| \gtrsim\left|\frac{\tau_{\mathrm{ASF}}}{\rho_{0} f_{0}}\right|=\Psi_{\mathrm{ASF}} .
$$

This states that in order to form water on the continental shelf that is dense enough to sink to the deep ocean bed, the overturning must be stronger than the wind-driven Ekman overturning circulation in the ASF. Note that if condition (28) is satisfied then the deepest isotherms originating from $\tilde{y}=0$ do not form a $\mathrm{V}$ as they cross the continental slope, as explained in the previous subsection. These results are summarized in section 7 .

\section{Sensitivity to surface wind stress}

We now investigate the sensitivity of our eddyresolving simulations and residual-mean model to changes in the surface wind stress. We define three sets of sensitivity experiments by varying the wind stress parameters $\tau_{\mathrm{ACC}}$ and $\tau_{\mathrm{ASF}}$ about their reference values.

\section{a. Zonal transport}

The zonal transport is the most fundamental measure of the ACC and ASF, which are clearly identified in 

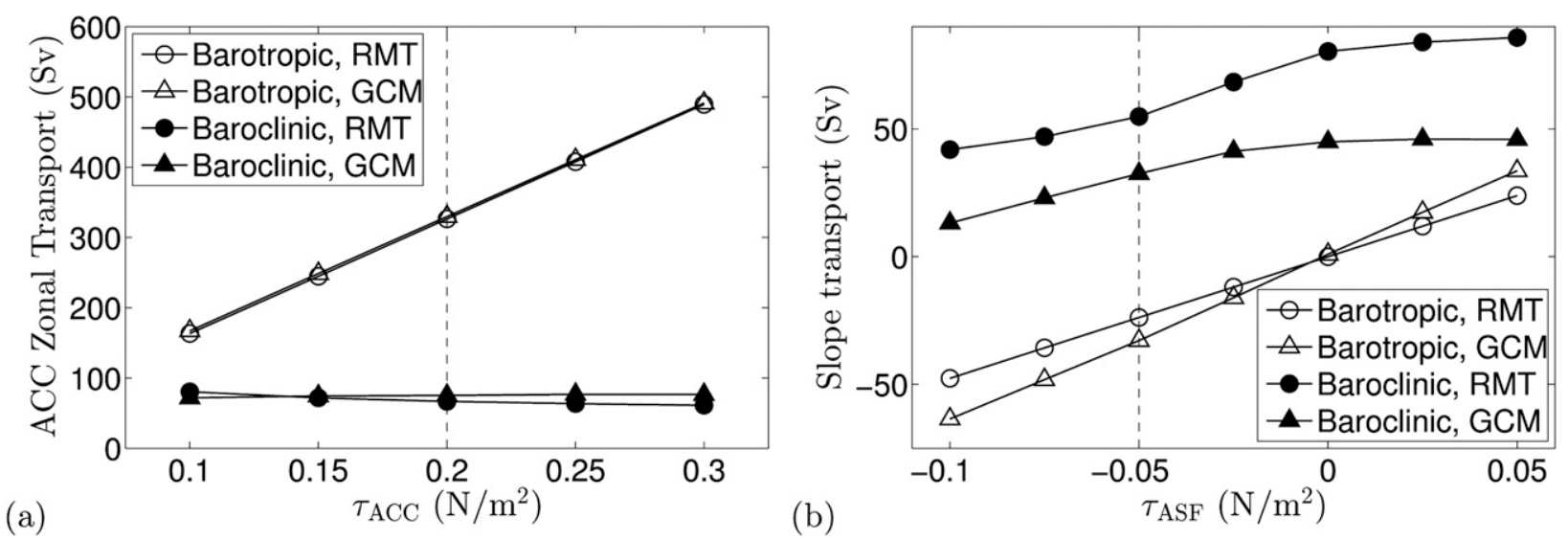

FIG. 10. Sensitivity of the zonal transport (a) in the ACC $(800 \leq y \leq 1900 \mathrm{~km})$ and (b) over the slope $(200 \leq y \leq 800 \mathrm{~km})$ to changes in the westerly wind strength $\tau_{\mathrm{ACC}}$ and easterly wind strength $\tau_{\mathrm{ASF}}$ respectively. The vertical dashed lines indicate our reference simulation from section 2 .

the mean zonal velocity $\bar{u}$ for our reference simulation, shown in Fig. 2. We determine the zonal transport in our residual-mean solutions from the temperature stratification via thermal-wind balance,

$$
\bar{u}=\bar{u}_{b}-\frac{\alpha g}{f_{0}} \int_{-h}^{z} \frac{\partial \bar{\theta}}{\partial y} d z^{\prime} \quad \text { and } \quad \bar{u}_{b}=\frac{\tau}{\rho_{0} r_{b}} .
$$

Here we have assumed that at each latitude, the mean basal velocity $\bar{u}_{b}=\left.\bar{u}\right|_{z=-h}$ extracts all momentum input by surface wind stress via bottom friction. It would be more realistic to prescribe $\left.\bar{u}\right|_{z=-h+H_{b}}$ and assume that the wind-input momentum is removed by viscous stress in the bottom boundary layer of thickness $H_{b}$, but (29) is a good approximation.

In Fig. 10a we plot the response of the zonal transport in the ACC, defined as $800 \leq y \leq 1900 \mathrm{~km}$, to changes in the midlatitude westerly wind strength $\tau_{\text {ACC }}$. The barotropic component is simply the basal velocity, and is slightly smaller in the RMT solutions than in our simulations. The baroclinic transport in our simulations is almost completely independent of $\tau_{\mathrm{ACC}}$, indicating that our model ACC is in an "eddy saturated" regime (Meredith et al. 2011). This is partially a consequence of the thermal relaxation at the southern and northern boundaries, which inevitably leads to a meridional temperature gradient across the domain. In RMT, the isotherm slope is given by (21). In the ACC $K \equiv K_{0}$ is constant and $\bar{\psi}$ typically dominates $\psi^{\dagger}$, so $K_{0}$ must vary approximately linearly with $\tau_{\mathrm{ACC}}$ in order to leave $s_{\theta}$ unchanged. We therefore prescribe the eddy diffusivity scale $K_{0}$ in (17) as

$$
K_{0}=K_{\text {ref }} \frac{\tau_{\mathrm{ACC}}}{\tau_{\text {ref }}},
$$

where $K_{\text {ref }}=2000 \mathrm{~m}^{2} \mathrm{~s}^{-1}$ and $\tau_{\text {ref }}=0.2 \mathrm{~N} \mathrm{~m}^{-2}$. The resulting RMT-predicted stratification yields a baroclinic zonal velocity that agrees very closely with our simulations, as shown in Fig. 10a. We obtained equivalent RMT solutions that hold $K_{0}$ fixed across all values of $\tau_{\mathrm{ACC}}$, and found that the baroclinic transport varied by around $40 \mathrm{~Sv}$ between $\tau_{\mathrm{ASF}}=0.1 \mathrm{~N} \mathrm{~m}^{-2}$ and $\tau_{\mathrm{ASF}}=$ $0.3 \mathrm{~N} \mathrm{~m}^{-2}$.

In Fig. 10b we plot the response of the zonal transport over the continental slope, defined as $200 \leq y \leq$ $800 \mathrm{~km}$, to changes in the polar easterly wind strength $\tau_{\mathrm{ACC}}$. The net transport is close to zero in our reference simulation because of the surface-intensified eastward jets surrounding the ASF, visible in Fig. 2. In this case the RMT barotropic velocity is consistently around $25 \%$ smaller than the basal velocity in our simulations. This is due to a net northward flux of zonal momentum from the slope to the ACC, resulting in the basal velocities in both regions exceeding that predicted by (29). The discrepancy is more noticeable in Fig. 10b because of the smaller volume of the slope region. The baroclinic slope transports follow qualitatively similar patterns, but RMT consistently predicts a transport that is $30-50 \mathrm{~Sv}$ too large. This arises because the residual-mean solutions tend to form sharper temperature gradients close to the topography at the shelf break, resulting in a larger positive zonal velocity throughout the water column above. For $\tau_{\mathrm{ASF}} \geq 0$ the baroclinic transport is insensitive to the wind stress, and the stratification and overturning closely resemble those shown in Fig. 11.

\section{b. Overturning circulation}

The MOC in our eddy-resolving simulations has a broadly similar presentation to that shown in Fig. $3 b$ for all parameter combinations considered here. We quantify 

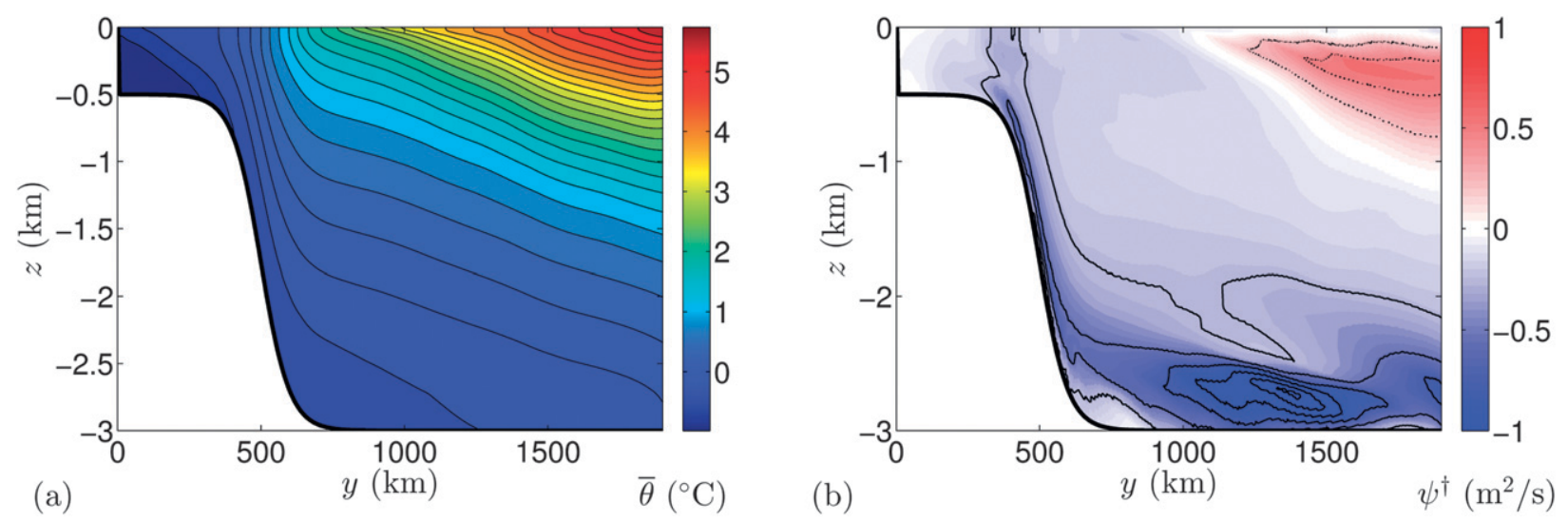

FIG. 11. As in Fig. 3, but for $\tau_{\text {ASF }}=0$.

the strength of the upper overturning cell following Abernathey et al. (2011), defining $\Psi_{\text {upper }}$ as the largest value of $\psi^{\dagger}$ anywhere in the domain. We quantify the lower overturning cell following Stewart and Thompson (2012); we restrict our attention to streamlines that extend from the shelf cooling region at $y=200 \mathrm{~km}$ to $y=1900 \mathrm{~km}$. We then define $\Psi_{\text {lower }}$ as the largest negative value of $\psi^{\dagger}$ over all such streamlines. This excludes the influence of the deep recirculation visible in Fig. 3b, and measures the net transport from the southernmost to the northernmost edges of the model Southern Ocean.

Figure 12a shows the response of $\Psi_{\text {upper }}$ and $\Psi_{\text {lower }}$ to changes in $\tau_{\mathrm{ACC}}$, where again $K_{0}$ is prescribed following (30). The upper overturning cell exhibits a modest sensitivity to changes in the midlatitude westerlies in our simulations, with a least squares slope of $1.39 \mathrm{~m}^{4} \mathrm{~s}^{-1} \mathrm{~N}^{-1}$. Abernathey et al. (2011) obtained a somewhat larger sensitivity of $2.6 \mathrm{~m}^{4} \mathrm{~s}^{-1} \mathrm{~N}^{-1}$ using fixed surface buoyancy fluxes. This discrepancy may be due to the range of temperatures spanned by the upper cell, determined by the northern boundary condition, and the relative positions of the wind stress and surface heat flux maxima. The simulated stratification and overturning over the continental shelf and slope are not modified by changes in $\tau_{\mathrm{ACC}}$, so $\Psi_{\text {lower }}$ is relatively insensitive. In RMT $\Psi_{\text {upper }}$ has a similar magnitude and increases with $\tau_{\text {ACC }}$, but the least squares sensitivity of $0.78 \mathrm{~m}^{4} \mathrm{~s}^{-1} \mathrm{~N}^{-1}$ is only around half of that in the simulations. This discrepancy arises because the upper overturning is set at the surface by (14c), and the surface heat flux $\Theta_{m}(y)$ is prescribed by (3) over all of our RMT solutions. Thus changes in $\Psi_{\text {upper }}$ are due entirely to the surface temperature gradient, which differs visibly in Figs. $3 \mathrm{a}$ and 8. A more sophisticated parameterization of the ACC eddy diffusivity $K$ is required to capture the surface temperature gradient accurately, and thus predict the sensitivity of $\Psi_{\text {upper }}$ to changes in $\tau_{\text {ACC. }}$.
Figure $12 \mathrm{~b}$ shows that in both our simulations and in our RMT, $\Psi_{\text {upper }}$ is insensitive changes in $\tau_{\text {ASF }}$.

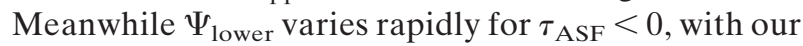
GCM and RMT predicting least squares sensitivities of $5.97 \mathrm{~m}^{4} \mathrm{~s}^{-1} \mathrm{~N}^{-1}$ and $6.44 \mathrm{~m}^{4} \mathrm{~s}^{-1} \mathrm{~N}^{-1}$, respectively. We also plot the asymptotically derived constraint on $\Psi_{\text {lower }}$
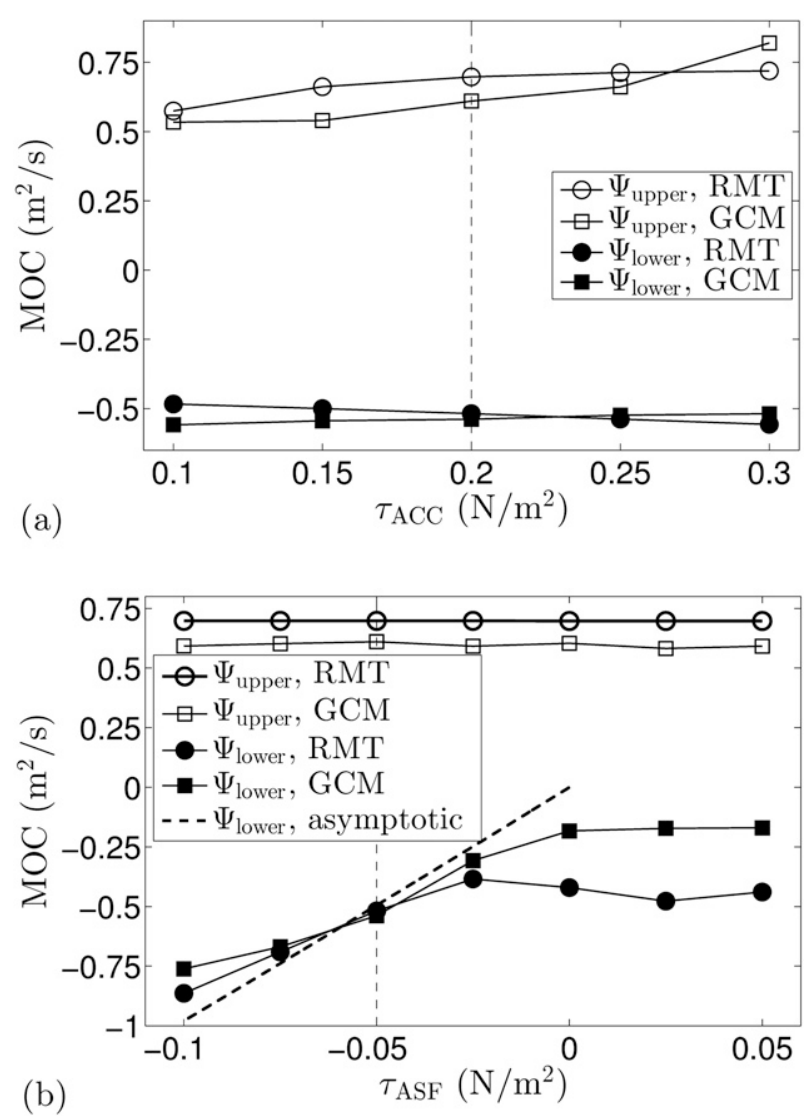

FIG. 12. Sensitivity of the overturning circulation to changes in (a) the westerly wind strength $\tau_{\mathrm{ACC}}$ and (b) the easterly wind strength $\tau_{\text {ASF }}$. 


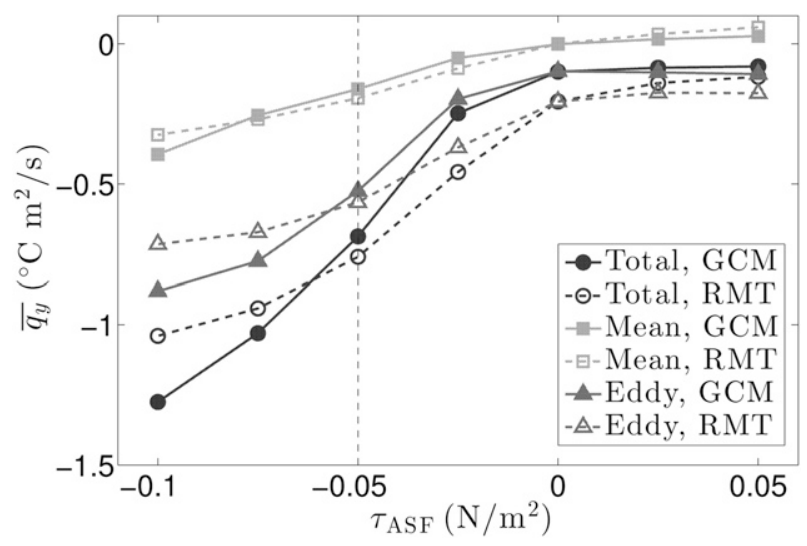

FIG. 13. Sensitivity of the meridional heat flux across the shelf break $(y=300 \mathrm{~km})$ to changes in the wind stress $\tau_{\text {ASF }}$ over the continental slope.

from section 5, obtained by setting the right-hand side of (26) to zero. This constraint predicts a stronger sensitivity of $9.80 \mathrm{~m}^{4} \mathrm{~s}^{-1} \mathrm{~N}^{-1}$, very close to that of the wind-driven mean overturning $\bar{\psi}$ over the slope. This discrepancy is due to the neglect of diapycnal mixing in (26), which intensifies the deep overturning close to the ocean bed. For $\tau_{\mathrm{ASF}} \geq 0$ the deep overturning is largely driven by surface heat flux, as shown in Fig. 11. In this regime the stratification is insensitive to $\tau_{\mathrm{ASF}}$ due to eddy saturation over the continental slope, as shown in Fig. 10b, and the surface heat flux $\Theta_{m}$ is fixed. Thus $\Psi_{\text {lower }}$ also becomes insensitive to $\tau_{\mathrm{ASF}}$. Our RMT solutions substantially overpredict the magnitude of $\Psi_{\text {lower }}$ in this regime because the simulated isotherms on the shelf become too steep to be described by a constant eddy diffusivity $K_{0}$.

\section{c. Cross-shelf heat flux}

Figure 13 shows the zonally averaged cross-shelf heat flux $\overline{q_{y}}$ for various wind strengths $\tau_{\mathrm{ASF}}$ over the continental slope (cf. Fig. 4). As in our reference simulation, the eddy component of $\overline{q_{y}}$ is much larger than the mean component for all values of $\tau_{\mathrm{ASF}}$. It is unsurprising that the eddy heat flux follows a similar pattern to $\Psi_{\text {lower }}$ because the overturning on the shelf is due to the exchange of warm and cold eddies across the shelf break, as shown in Fig. 4. As $\bar{v}$ is confined to the surface mixed layer and bottom boundary, the mean heat flux may be expected to be approximately proportional to $\tau_{\mathrm{ASF}}$ and to the temperature difference between the surface and the shelf. The shelf waters become weakly stratified for $\tau_{\mathrm{ASF}} \geq 0$, as shown in Fig. 11, resulting in only a small northward heat flux in this regime. Thus the net heat flux varies more rapidly with $\tau_{\mathrm{ASF}}<0$ than with $\tau_{\mathrm{ASF}} \geq 0$. This is consistent with the hypothesis that warm CDW inflow to the Ross and Amundsen Seas is controlled by seasonal changes in the offshore easterlies (Thoma et al.
2010; Dinniman et al. 2011), but seems to contradict the idea that changes in offshore westerly wind stress control CDW intrusions into the Amundsen Sea (Thoma et al. 2008).

\section{Conclusions}

In this study we have developed eddy-resolving simulations and a residual-mean theory for an idealized sector of the Southern Ocean, including a representation of the Antarctic continental shelf and slope. Our theory includes boundary conditions for the surface mixed layer, the shelf cooling region, the bottom boundary layer, and the northern relaxation region. Our simulations exhibit suppression of baroclinic instability over the continental slope, and strong diapycnal mixing close to the ocean bed. These effects could not be predicted a priori and are critical features of the dynamics in this system, so our theory incorporates simple parameterizations for the isopycnal and diapycnal diffusivities $K$ and $\kappa$ based on our reference simulation. Away from these regions $K$ and $\kappa$ are held constant, yet the mean stratification and overturning in Fig. 8 agrees convincingly with those diagnosed from of our reference simulation in Fig. 3. This study reinforces the importance of resolving mesoscale eddies over the continental slope, or at least employing a careful parameterization of their transport. The most frequently used parameterizations of $K$ would not capture the behavior of the eddies in our simulations (see Isachsen 2011), and may be expected to produce a qualitatively different stratification, more closely resembling Fig. 9a than Figs. 3a or 8a. Indeed, Fig. 9a suggests that it is the suppression of baroclinic eddies over the continental slope that permits our model ocean to form its densest water on the continental shelf.

Residual-mean theory intuitively explains the sharp $\mathrm{V}$-shaped isopycnal structure that characterizes the ASF in bottom water-forming regions of the Antarctic continental shelf (e.g., Thompson and Heywood 2008) as the result of suppression of baroclinic instability over the continental slope. The theory also accounts for stretches of the continental shelf where little or no dense water is formed, and the shoreward side of the $\mathrm{V}$ is absent (Whitworth et al. 1998), as illustrated in Fig. 9b. Both our residual-mean solutions and our eddy-resolving simulations predict that the strength of the deep overturning circulation is constrained to be at least comparable in strength to the Ekman transport driven by the polar easterly winds. In section 5 we showed via (28) that this is approximately equivalent to requiring that the densest isopycnals emanating from the continental shelf do not form a $\mathrm{V}$ shape, but rather sink down the continental slope and outcrop at the ocean bed. The existence 


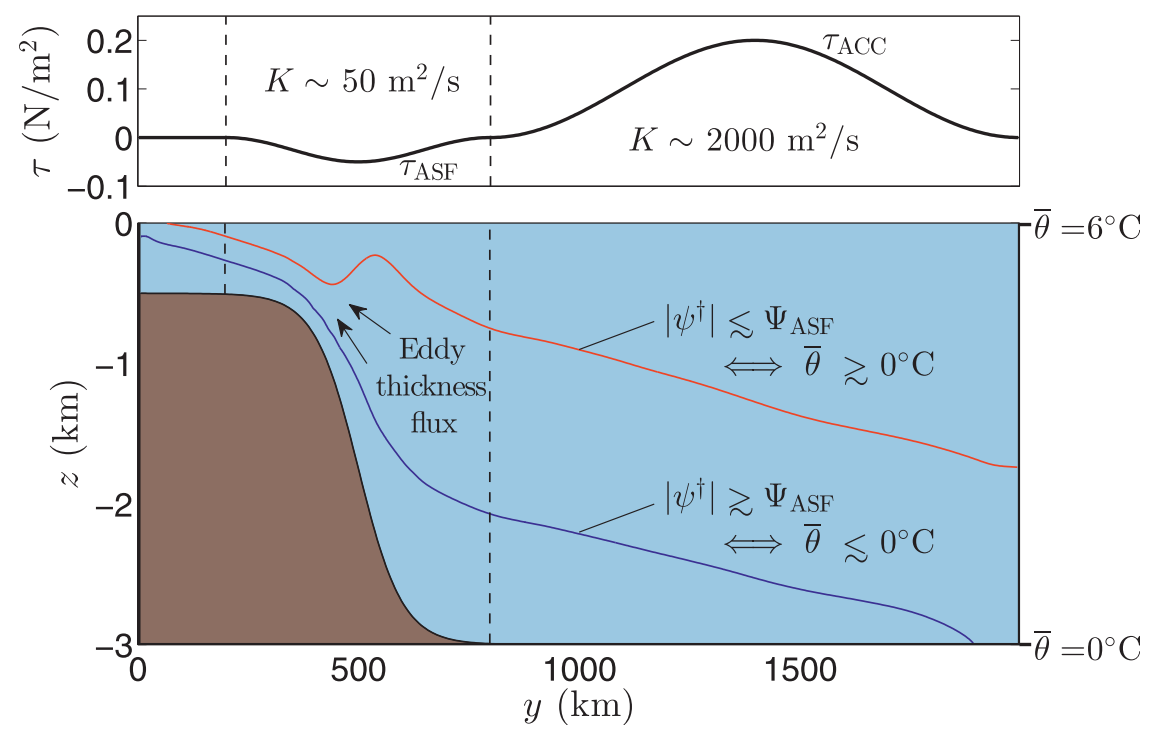

FIG. 14. A schematic illustrating the formation of a V-shaped ASF and sensitivity of the deep MOC to the easterly driven mean overturning $\Psi_{\mathrm{ASF}}$. The ocean interior is approximately adiabatic, so $\psi^{\dagger} \approx \psi^{\dagger}(\bar{\theta})$. The red isotherm forms a V-shaped ASF over the continental slope because the winddriven Ekman pumping deepens/shoals isotherms on the poleward/equatorward side of the ASF. The blue isotherm must instead plunge down to the ocean bed to supply a sufficiently large poleward eddy thickness flux over the continental slope, where $K$ is greatly reduced because of suppression of baroclinic instability. Thus if denser water is to emanate from the shelf than exists at the northern boundary, the deep MOC $\psi^{\dagger}$ must be at least comparable to $\Psi_{\mathrm{ASF}}$ on those isotherms.

of such isopycnals is necessary to support denser waters on the shelf than at the northern boundary. These key results are illustrated schematically in Fig. 14.

There are a number of potential improvements to the admittedly idealized model described in this study, which would help to generate more realistic flows. For example, zonal variations in the bottom topography constitute an important omission from our model configuration. Submarine canyons may support geostrophic mean cross-slope transports or turbulent down-slope currents, providing an alternative route for AABW to escape the continental shelf, and deep-ocean ridges may support geostrophic transport of AABW across the ACC (e.g., Naveira Garabato et al. 2002). Such features could substantially alter the influence of the polar easterlies on the deep overturning cell, and require further investigation. Another desirable addition would be the dependence of density on salinity, which is particularly pronounced over the Antarctic continental shelf because of nonlinearities in the equation of state (Gill 1973). With sufficient resolution the importance of the thermobaric effect on dense water formation and export could also be explored. Incorporation of sea ice and ice sheets adds considerable complexity but would ultimately provide further insight into the dynamical processes governing cross-shelf exchange. We purposefully kept our residual-mean model as simple as possible to permit a fair comparison with our simulations, and there is certainly scope for improving our parameterizations of the eddy and diapycnal diffusivities. For example, the simulated eddy diffusivity $K$ is accurately described by the parameterization of Visbeck et al. (1997) everywhere except over the continental slope. While all of these aspects would entail significant modifications to the theory developed here, the success of RMT in reproducing the stratification and overturning in our simulations demonstrates its potential to explain complex dynamical features like export of AABW and the structure of the ASF.

Acknowledgments. A.L.S.'s and A.F.T.'s research was supported by the California Institute of Technology and NSF Award OCE-1235488. The simulations presented herein were conducted using the CITerra computing cluster in the Division of Geological and Planetary Sciences at the California Institute of Technology, and the authors thank the CITerra technicians for facilitating this work. The authors gratefully acknowledge the modeling efforts of the MITgcm team. The authors thank Dimitris Menemenlis, Christopher Wolfe, Rick Salmon, Paul Dellar and Mike Dinniman for useful discussions. The authors thank two anonymous reviewers for constructive criticisms that improved the original draft of this manuscript. 


\section{APPENDIX}

\section{Boundary Conditions for Residual-Mean Theory}

\section{a. The surface mixed layer}

As shown in in Fig. 5, we approximate the surface mixed layer by assuming that the $\bar{\theta}$ is independent of depth, $\bar{\theta}=\bar{\theta}_{m}(y)$ (Marshall and Radko 2003). Under this assumption (8) becomes

$$
-\frac{\partial \psi^{\dagger}}{\partial \tilde{z}} \frac{d \bar{\theta}_{m}}{d \tilde{y}}=\frac{\partial \Theta}{\partial \tilde{z}}+\frac{\partial}{\partial \tilde{y}}\left(\kappa \frac{d \bar{\theta}_{m}}{d \tilde{y}}\right),
$$

where we have written the heat source $S_{\theta}$ as the vertical divergence of a heat flux $\Theta$. We denote the surface heat flux as $\left.\Theta\right|_{\tilde{z}=H_{m}}=\Theta_{m}(\tilde{y})=-Q(\tilde{y}) / \rho_{0} C_{p}$, where $C_{p}=4 \times$ $10^{3} \mathrm{~J} \mathrm{~K}^{-1} \mathrm{~kg}^{\underline{m} 1}$ is the specific heat capacity. We assume that all of the surface heat flux is absorbed in the mixed layer, so $\Theta=0$ at $\tilde{z}=0$. To obtain a boundary condition for $\bar{\psi}$, we integrate (A1) from $\tilde{z}=0$ to $\tilde{z}=H_{m}$ using $\psi^{\dagger}=$ 0 at $\tilde{z}=H_{m}$,

$\left.\psi^{\dagger}\right|_{\tilde{z}=0}=\left(\frac{d \bar{\theta}_{m}}{d \tilde{y}}\right)^{-1}\left[\Theta_{m}+\frac{d}{d \tilde{y}}\left(\frac{d \bar{\theta}_{m}}{d \tilde{y}} \int_{0}^{H_{m}} \kappa d \tilde{z}\right)\right]$.

Thus the residual water mass exchange between the ocean interior and the surface mixed layer is determined by the surface heat input and meridional temperature gradient.

\section{$b$. The shelf cooling region}

The water on the continental shelf is subject to direct thermal relaxation to a temperature of $\theta_{c}=-1^{\circ} \mathrm{C}$,

$$
S_{\theta}={\overline{-T^{-1}\left(\bar{\theta}^{t}-\theta_{c}\right)}}^{x}, \quad-L_{c} \leq \tilde{y} \leq 0,
$$

where the time scale $T$ is given by (2). This thermal relaxation results in a zonal asymmetry in the time-mean temperature of the shelf waters, visible in Fig. 1. To evaluate (A3) exactly, we would need to prescribe the three-dimensional temperature structure everywhere on the shelf. Instead we derive an approximate boundary condition for RMT under the assumption that the zonal variations of $\bar{\theta}^{t}$ are small compared to its meridional and vertical stratification. This amounts to replacing $\bar{\theta}^{t}$ with $\bar{\theta}$ in (A3), which may then be written as

$$
S_{\theta} \approx \frac{\left(\tilde{z}-H_{m}\right) \sqrt{\pi} L_{c}}{2 T_{c} H_{s} L_{x}} \exp \left[-\left(\frac{\tilde{y}+L_{c}}{1 / 2 L_{c}}\right)^{2}\right]\left(\bar{\theta}-\theta_{c}\right),
$$

Here we have used ${\overline{\exp \left\{-\left[x /(1 / 2) L_{c}\right]^{2}\right\}}}^{x} \approx \sqrt{\pi} L_{c} / 2 L_{x}$ to evaluate ${\overline{T^{-1}}}^{x}$ from (2), which holds because $L_{c} \ll L_{x}$.
We further assume that the isotherm slope is uniform in the cooling region, $s_{\theta} \equiv s_{c}=-\tilde{H}_{s} / L_{c}$, such that the coldest isotherm connects $\left(0,-\tilde{H}_{s}\right)$ to $\left(-L_{c}, 0\right)$, as shown in Fig. 5. The shelf temperature may therefore be written as

$$
\bar{\theta}(\tilde{y}, \tilde{z})=\bar{\theta}\left(0, \tilde{z}-s_{c} \tilde{y}\right), \quad-L_{c} \leq \tilde{y} \leq 0,
$$

so both $\bar{\theta}$ and $\partial_{\bar{z}} \bar{\theta}$ are constant along each isotherm. This assumption may seem restrictive, but in fact (A5) is similar to the mean shelf temperature in our simulations. In sections 5-6 we show that the resulting boundary streamfunction $\psi^{\dagger}(0, \tilde{z})$ agrees well with the simulated overturning circulation over a range of wind stress parameters.

In general, given $S_{\theta}(\tilde{y}, \tilde{z})$ and $\bar{\theta}(\tilde{y}, \tilde{z})$ everywhere in $\tilde{y} \in$ $\left(-L_{c}, 0\right)$ and $\tilde{z} \in\left(-\tilde{H}_{s}, H_{m}\right)$, we may determine the residual streamfunction $\psi^{\dagger}\left(0, \tilde{z}_{0}\right)$ at any depth $\tilde{z}_{0}$. We rewrite (8) as a derivative of $\psi^{\dagger}$ along the contour $\bar{\theta}=\bar{\theta}\left(0, \tilde{z}_{0}\right)$ (e.g., Karsten and Marshall 2002),

$$
\frac{\partial \psi^{\dagger}}{\partial l}=\frac{S_{\theta}+\nabla \cdot(\kappa \nabla \bar{\theta})}{\sqrt{\left(\partial_{\tilde{y}} \bar{\theta}\right)^{2}+\left(\partial_{\tilde{z}} \bar{\theta}\right)^{2}}},
$$

where $l$ is an along-contour coordinate. Scaling analysis suggests that the direct cooling $S_{\theta}$ should dominate diapycnal mixing, and our simulations confirm that this is the case. We therefore neglect the term proportional to $\kappa$ in (A6). We also neglect the portion of each isotherm that lies within the mixed layer, where $\left(\tilde{z}-H_{m}\right) / H_{s} \ll 1$ is small and so the cooling $S_{\theta}$ is weak. Prescribing the temperature of the shelf waters using (A5), (A6) may be written as

$$
\psi^{\dagger}\left(0, \tilde{z}_{0}\right)=\left.\int_{\tilde{y}_{0}}^{0} d \tilde{y} \frac{S_{\theta}}{\partial_{\tilde{z}} \bar{\theta}}\right|_{\tilde{z}=\tilde{z}_{0}+s_{c} \tilde{y}} .
$$

Here $\tilde{y}_{0}=L_{c} \tilde{z}_{0} / \tilde{H}_{s}$ is the position at which the isotherm $\bar{\theta}=\bar{\theta}\left(0, \tilde{z}_{0}\right)$ meets the mixed layer at $\tilde{z}=0$. By assumption (A5), $\bar{\theta}\left(\tilde{y}, s_{c} \tilde{y}\right)=\bar{\theta}\left(0, \tilde{z}_{0}\right)$ and $\partial_{\tilde{z}} \bar{\theta}\left(\tilde{y}, s_{c} \tilde{y}\right)=\partial_{\tilde{z}} \bar{\theta}\left(0, \tilde{z}_{0}\right)$ are constant along each isotherm, so we may integrate (A7) exactly to obtain

$$
\psi^{\dagger}(0, \tilde{z})=V_{c} \frac{\left[\bar{\theta}(0, \tilde{z})-\theta_{c}\right]}{\partial_{\tilde{z}} \bar{\theta}(0, \tilde{z})} I(\tilde{z}) \quad \text { and } \quad V_{c}=\frac{\sqrt{\pi} L_{c}^{2} \tilde{H}_{s}}{2 T_{c} H_{s} L_{x}}
$$

Here $V_{c}$ is a velocity scale for the heat flux across $\tilde{y}=0$, whose vertical structure is described by 


$$
\begin{aligned}
I(\tilde{z})= & \frac{1}{8}\left\{\exp (-4)-\exp \left[-4\left(1+\tilde{z} / \tilde{H}_{s}\right)^{2}\right]\right\} \\
& +\frac{\sqrt{\pi}}{4 \tilde{H}_{s}}\left(\tilde{z}+\tilde{H}_{s}-H_{m}\right)\left\{\operatorname{erf}(2)-\operatorname{erf}\left[2\left(1+\tilde{z} / \tilde{H}_{s}\right)\right]\right\} .
\end{aligned}
$$

Note that (12) still requires that $\psi^{\dagger}[0,-\tilde{h}(0)]=0$ must be satisfied at the bottom boundary.

\section{c. The bottom boundary layer}

To satisfy the no-flux boundary condition (12), the wind-driven mean overturning $\bar{\psi}$ must adjust from its interior form (9) to zero at $\tilde{z}=-\tilde{h}(\tilde{y})$, where $\tilde{h}=$ $h-H_{m}$. In the real ocean this is achieved via a bottom Ekman layer, which may be described analytically under the assumption of a negligible horizontal buoyancy gradient (e.g., Pedlosky 1987; Vallis 2006). The bottom Ekman layer is not resolved in our simulations, where instead $\bar{\psi}$ is closed via a mean meridional transport through the lowermost grid cells. We employ a simple representation of the bottom boundary layer similar to that of Ferrari et al. (2008),

$$
\bar{\psi}=-\frac{\tau(\tilde{y})}{\rho_{0} f_{0}} F_{b}(\tilde{z})
$$

where

$$
F_{b}(\tilde{z})= \begin{cases}3\left(\frac{\tilde{z}_{h}}{H_{b}}\right)^{2}-2\left(\frac{\tilde{z}_{h}}{H_{b}}\right)^{3}, & 0 \leq \tilde{z}_{h} \leq H_{b}, \\ 1, & H_{b} \leq \tilde{z}_{h} \leq h .\end{cases}
$$

Here $\tilde{z}_{h}=\tilde{z}+\tilde{h}$ is the height above the topography. This form ensures that $\bar{\psi}$ is continuous and differentiable at $\tilde{z}=-\tilde{h}+H_{b}$, and that $\bar{v}=0$ at $\tilde{z}=-\tilde{h}$. As $\psi^{\star}$ describes the mass transport due to geostrophic eddies, it should also vanish in the ocean's bottom Ekman layer. We therefore prescribe

$$
\psi^{\star}=K F_{b}(\tilde{z}) s_{\theta},
$$

which effectively reduces the eddy diffusivity $K$ to zero in the bottom boundary layer. To satisfy all parts of (12), we also require

$$
\frac{\partial \bar{\theta}}{\partial \tilde{z}}=s_{b} \frac{\partial \bar{\theta}}{\partial \tilde{y}}, \quad \text { on } \quad \tilde{z}=-\tilde{h}
$$

where $s_{b}=-d_{\tilde{y}} \tilde{h}$ is the slope of the bottom topography.

\section{d. The northern boundary}

In our simulations, the ocean temperature is relaxed over the northernmost $L_{n}=100 \mathrm{~km}$ of the domain to a prescribed exponential profile (5),

$$
S_{\theta}=-T_{r}^{-1} \frac{\tilde{y}-\tilde{L}_{y}+L_{n}}{L_{n}}\left[\bar{\theta}-\theta_{n}(\tilde{z})\right],
$$

for $\tilde{y} \geq \tilde{L}_{y}-L_{n}$, where $\tilde{L}_{y}=L_{y}-L_{c}$. The region $\tilde{y} \in$ $\left(\tilde{L}_{y}-L_{n}, \tilde{L}_{y}\right)$ may thus be included directly in the RMT solutions described in section 5. However, in practice $\bar{\theta}$ remains very close to $\theta_{n}$ in this region, so for simplicity we prescribe $\bar{\theta}\left(\tilde{L}_{y}-L_{n}, \tilde{z}\right)=\theta_{n}(\tilde{z})$.

\section{REFERENCES}

Abernathey, R., J. Marshall, and D. Ferreira, 2011: The dependence of Southern Ocean meridional overturning on wind stress. J. Phys. Oceanogr., 41, 2261-2278.

Andrews, D. G., J. R. Holton, and C. B. Leovy, 1987: Middle Atmosphere Dynamics. International Geophysics Series, Vol. 40, Academic Press, 489 pp.

Baines, P. G., 2009: A model for the structure of the Antarctic Slope Front. Deep-Sea Res. II, 56 (13-14), 859-873.

Chavanne, C. P., K. J. Heywood, K. W. Nicholls, and I. Fer, 2010: Observations of the Antarctic slope undercurrent in the southeastern Weddell Sea. Geophys. Res. Lett., 37, L13601, doi:10.1029/2010GL043603.

Dinniman, M. S., J. M. Klinck, and W. O. Smith, 2011: A model study of Circumpolar Deep Water on the West Antarctic Peninsula and Ross Sea continental shelves. Deep-Sea Res. II, $\mathbf{5 8}, 1508-1523$.

Döös, K., and D. J. Webb, 1994: The Deacon cell and the other meridional cells of the Southern Ocean. J. Phys. Oceanogr., 24, 429-429.

Ferrari, R., J. C. McWilliams, V. M. Canuto, and M. Dubovikov, 2008: Parameterization of eddy fluxes near oceanic boundaries. J. Climate, 21, 2770-2789.

Ferreira, D., J. Marshall, and P. Heimbach, 2005: Estimating eddy stresses by fitting dynamics to observations using a residualmean ocean circulation model and its adjoint. J. Phys. Oceanogr., 35, 1891-1910.

Garabato, A. C. N., K. L. Polzin, B. A. King, K. J. Heywood, and M. Visbeck, 2004: Widespread intense turbulent mixing in the southern ocean. Science, 303, 210-213.

Gent, P. R., and J. C. McWilliams, 1990: Isopycnal mixing in ocean circulation models. J. Phys. Oceanogr., 20, 150-155.

Gill, A. E., 1973: Circulation and bottom water production in the Weddell Sea. Deep-Sea Res., 20,111-140.

Gordon, A. L., 2009: Bottom water formation. Ocean Currents, J. H. Steele, S. A. Thorpe, and K. K. Turekian, Eds., Associated Press, 263-269.

Hallberg, R., and A. Gnanadesikan, 2006: The role of eddies in determining the structure and response of the wind-driven Southern Hemisphere overturning: Results from the Modeling Eddies in the Southern Ocean (MESO) project. J. Phys. Oceanogr., 36, 2232-2252.

Hill, C., D. Ferreira, J. M. Campin, J. Marshall, R. Abernathey, and N. Barrier, 2012: Controlling spurious diapycnal mixing in 
eddy-resolving height-coordinate ocean models-Insights from virtual deliberate tracer release experiments. Ocean Modell., 45, 14-26.

Hofmann, M., and M. A. M. Maqueda, 2011: The response of Southern Ocean eddies to increased midlatitude westerlies: A non-eddy resolving model study. Geophys. Res. Lett., 38, L03605, doi:10.1029/2010GL045972.

Hogg, A. M. C., M. P. Meredith, J. R. Blundell, and C. Wilson, 2008: Eddy heat flux in the Southern Ocean: Response to variable wind forcing. J. Climate, 21, 608-620.

Isachsen, P. E., 2011: Baroclinic instability and eddy tracer transport across sloping bottom topography: How well does a modified Eady model do in primitive equation simulations? Ocean Modell., 39, 183-199.

Ito, T., and J. Marshall, 2008: Control of lower-limb overturning circulation in the Southern Ocean by diapycnal mixing and mesoscale eddy transfer. J. Phys. Oceanogr., 38, 2832-2845.

Jacobs, S. S., 1991: On the nature and significance of the Antarctic Slope Front. Mar. Chem., 35, 9-24.

Karsten, R. H., and J. Marshall, 2002: Constructing the residual circulation of the ACC from observations. J. Phys. Oceanogr., 32, 3315-3327.

Kitoh, A., S. Murakami, and H. Koide, 2001: A simulation of the Last Glacial Maximum with a coupled atmosphere-ocean GCM. Geophys. Res. Lett., 28, 2221-2224.

Krinner, G., and C. Genthon, 1998: GCM simulations of the Last Glacial Maximum surface climate of Greenland and Antarctica. Climate Dyn., 14, 741-758.

Kuhlbrodt, T., A. Griesel, M. Montoya, A. Levermann, M. Hofmann, and S. Rahmstorf, 2007: On the driving processes of the Atlantic meridional overturning circulation. Rev. Geophys., 45, RG2001, doi:10.1029/2004RG000166.

Kuo, A., R. A. Plumb, and J. Marshall, 2005: Transformed Eulerianmean theory. Part II: Potential vorticity homogenization and the equilibrium of a wind-and buoyancy-driven zonal flow. J. Phys. Oceanogr., 35, 175-187.

Kurganov, A., and E. Tadmor, 2000: New high-resolution central schemes for nonlinear conservation laws and convectiondiffusion equations. J. Comput. Phys., 160, 241-282.

Large, W. G., and S. G. Yeager, 2009: The global climatology of an interannually varying air-sea flux data set. Climate Dyn., 33, 341-364.

— J J. C. McWilliams, and S. C. Doney, 1994: Oceanic vertical mixing: A review and a model with a nonlocal boundary layer parameterization. Rev. Geophys., 32, 363-404.

Lumpkin, R., and K. Speer, 2007: Global ocean meridional overturning. J. Phys. Oceanogr., 37, 2550-2562.

Lynch-Stieglitz, J., and Coauthors, 2007: Atlantic meridional overturning circulation during the last glacial maximum. Science, 316, 66-69.

Marshall, J., and T. Radko, 2003: Residual-mean solutions for the Antarctic Circumpolar Current and its associated overturning circulation. J. Phys. Oceanogr., 33, 2341-2354.

— onal overturning of the Southern Ocean. Prog. Oceanogr., 70 (2-4), 331-345.

—, and K. Speer, 2012: Closure of the meridional overturning circulation through Southern Ocean upwelling. Nat. Geosci., 5, 171-180.

, A. Adcroft, C. Hill, L. Perelman, and C. Heisey, 1997a: A finite-volume, incompressible Navier Stokes model for studies of the ocean on parallel computers. J. Geophys. Res., 102 (C3), $5753-5766$.
— C. Hill, L. Perelman, and A. Adcroft, 1997b: Hydrostatic, quasi-hydrostatic, and nonhydrostatic ocean modeling. J. Geophys. Res., 102 (C3), 5733-5752.

McIntosh, P. C., and T. J. McDougall, 1996: Isopycnal averaging and the residual mean circulation. J. Phys. Oceanogr., 26, 16551660.

Meredith, M. P., A. C. Naveira Garabato, A. M. C. Hogg, and R. Farneti, 2011: Sensitivity of the overturning circulation in the Southern Ocean to decadal changes in wind forcing. J. Climate, 25, 99-110.

Muench, R. D., and A. L. Gordon, 1995: Circulation and transport of water along the western Weddell Sea margin. J. Geophys. Res., 100 (C9), 18503-18515.

Naveira Garabato, A. C., E. L. McDonagh, D. P. Stevens, K. J. Heywood, and R. J. Sanders, 2002: On the export of Antarctic bottom water from the Weddell Sea. Deep-Sea Res. II, 49, 4715-4742.

Nikurashin, M., and G. Vallis, 2011: A theory of deep stratification and overturning circulation in the ocean. J. Phys. Oceanogr., 41, 485-502.

— and - 2012: A theory of the interhemispheric meridional overturning circulation and associated stratification. J. Phys. Oceanogr., 42, 1652-1667.

Nøst, O. A., M. Biuw, V. Tverberg, C. Lydersen, T. Hattermann, Q. Zhou, L. H. Smedsrud, and K. M. Kovacs, 2011: Eddy overturning of the Antarctic Slope Front controls glacial melting in the Eastern Weddell Sea. J. Geophys. Res., 116, C11014, doi:10.1029/2011JC006965.

Nowlin, W. D., Jr., and J. M. Klinck, 1986: The physics of the Antarctic circumpolar current. Rev. Geophys., 24, 469-491.

Olbers, D., and M. Visbeck, 2005: A model of the zonally averaged stratification and overturning in the Southern Ocean. J. Phys. Oceanogr., 35, 1190-1205.

Orsi, A. H., and C. L. Wiederwohl, 2009: A recount of Ross Sea waters. Deep-Sea Res. II, 56 (13-14), 778-795.

_ , S. S. Jacobs, A. L. Gordon, and M. Visbeck, 2001: Cooling and ventilating the abyssal ocean. Geophys. Res. Lett., 28 , 2923-2926.

Ou, H. W., 2007: Watermass properties of the Antarctic Slope Front: A simple model. J. Phys. Oceanogr., 37, 50-59.

Pavec, M., X. Carton, and G. Swaters, 2005: Baroclinic instability of frontal geostrophic currents over a slope. J. Phys. Oceanogr., 35, 911-918.

Pedlosky, J., 1987: Geophysical Fluid Dynamics. 2nd ed. SpringerVerlag, 710 pp.

Pennel, R., A. Stegner, and K. Béranger, 2012: Shelf impact on buoyant coastal current instabilities. J. Phys. Oceanogr., 42, 39-61.

Plumb, R. A., and R. Ferrari, 2005: Transformed Eulerian-mean theory. Part I: Nonquasigeostrophic theory for eddies on a zonal-mean flow. J. Phys. Oceanogr., 35, 165-174.

Polvani, L. M., D. W. Waugh, G. J. P. Correa, and S. W. Son, 2011: Stratospheric ozone depletion: The main driver of twentiethcentury atmospheric circulation changes in the Southern Hemisphere. J. Climate, 24, 795-812.

Radko, T., and J. Marshall, 2006: The Antarctic Circumpolar Current in three dimensions. J. Phys. Oceanogr., 36, 651-669.

Russell, J. L., K. W. Dixon, A. Gnanadesikan, R. J. Stouffer, and J. R. Toggweiler, 2006: The Southern Hemisphere westerlies in a warming world: Propping open the door to the deep ocean. J. Climate, 19, 6382-6390.

Saenko, O. A., A. S. Gupta, and P. Spence, 2011: On challenges in predicting bottom water transport in the Southern Ocean. J. Climate, 25, 1349-1356. 
Shindell, D. T., and G. A. Schmidt, 2004: Southern Hemisphere climate response to ozone changes and greenhouse gas increases. Geophys. Res. Lett., 31, L18209, doi:10.1029/2004GL020724.

Shu, C. W., and S. Osher, 1989: Efficient implementation of essentially non-oscillatory shock-capturing schemes, II. J. Comput. Phys., 83, 32-78.

Skinner, L., S. Fallon, C. Waelbroeck, E. Michel, and S. Barker, 2010: Ventilation of the deep Southern Ocean and deglacial $\mathrm{CO}_{2}$ rise. Science, 328, 1147-1151.

Son, S. W., and Coauthors, 2010: Impact of stratospheric ozone on Southern Hemisphere circulation change: A multimodel assessment. J. Geophys. Res., 115, D00M07, doi:10.1029/ 2010JD014271.

Speer, K., S. R. Rintoul, and B. Sloyan, 2000: The diabatic deacon cell. J. Phys. Oceanogr., 30, 3212-3222.

Stewart, A. L., and A. F. Thompson, 2012: Sensitivity of the ocean's deep overturning circulation to easterly Antarctic winds. Geophys. Res. Lett., 39, L18604, doi:10.1029/2012GL053099.

Stone, P. H., 1972: A simplified radiative-dynamical model for the static stability of rotating atmospheres. J. Atmos. Sci., 29, 405418.

Talley, L. D., J. L. Reid, and P. E. Robbins, 2003: Data-based meridional overturning streamfunctions for the global ocean. J. Climate, 16, 3213-3226.

Thoma, M., A. Jenkins, D. Holland, and S. Jacobs, 2008: Modelling circumpolar deep water intrusions on the Amundsen Sea continental shelf, Antarctica. Geophys. Res. Lett., 35, L18602, doi:10.1029/2008GL034939.

_ K. Grosfeld, K. Makinson, and M. A. Lange, 2010: Modelling the impact of ocean warming on melting and water masses of ice shelves in the Eastern Weddell Sea. Ocean Dyn., 60, 479-489.

Thompson, A. F., and K. J. Heywood, 2008: Frontal structure and transport in the northwestern Weddell Sea. Deep-Sea Res. I, 55, 1229-1251.

Thompson, D. W. J., and J. M. Wallace, 2000: Annular modes in the extratropical circulation. Part I: Month-to-month variability. J. Climate, 13, 1000-1016.

,-- , and G. Hegerl, 2000: Annular modes in the extratropical circulation. Part II: Trends. J. Climate, 13, 1018-1036.

Toggweiler, J. R., 2009: Shifting westerlies. Science, 323, 1434-1435.

— - J. Russell, and S. R. Carson, 2006: Midlatitude westerlies, atmospheric $\mathrm{CO}_{2}$, and climate change during the ice ages. Paleoceanography, 21, PA2005, doi:10.1029/2005PA001154.

Vallis, G. K., 2006: Atmospheric and Oceanic Fluid Dynamics: Fundamentals and Large-Scale Circulation. Cambridge University Press, 745 pp.

Visbeck, M., J. Marshall, T. Haine, and M. Spall, 1997: Specification of eddy transfer coefficients in coarse-resolution ocean circulation models. J. Phys. Oceanogr., 27, 381-402.

Whitworth, T., III, A. H. Orsi, S.-J. Kim, W. D. Nowlin Jr., and R. A. Locarnini, 1998: Water masses and mixing near the Antarctic slope front. Ocean, Ice, and Atmosphere: Interactions at the Antarctic Continental Margin, S. S. Jacobs and R. F. Weiss, Ed., Antarctic Research Series, Amer. Geophys. Union, 1-27.

Wolfe, C. L., and P. Cessi, 2011: The adiabatic pole-to-pole overturning circulation. J. Phys. Oceanogr., 41, 1795-1810.

Zhang, Y., J. Pedlosky, and G. R. Flierl, 2011: Cross-shelf and outof-bay transport driven by an open-ocean current. J. Phys. Oceanogr., 41, 2168-2186. 